\title{
Input to layer 1 of somatosensory cortex: Local input outweighs long-range and can modulate the activity of layer 1 interneurons
}

\section{Abbreviated title: Local versus long-range input to cortical layer 1}

\author{
Julia Ledderose ${ }^{1}$, Timothy A Zolnik ${ }^{1}$, Maria Toumazou ${ }^{1}$, Thorsten Trimbuch ${ }^{2}$, \\ Christian Rosenmund ${ }^{2,4}$, Britta J Eickholt ${ }^{3,4}$, Dieter Jaeger ${ }^{5}$, Matthew E Larkum ${ }^{1,4}$, \\ and Robert NS Sachdev ${ }^{1}$
}

1, Institute of Biology, Humboldt Universität zu Berlin Charitéplatz 1, Virchowweg 6, 10117 Berlin, Germany; 2, Institute of Neurophysiology, Charité - Universitätsmedizin Berlin, Charitéplatz 1, Virchowweg 6, 10117 Berlin, Germany; 3, Institute of Biochemistry, Charité Universitätsmedizin Berlin, Charitéplatz 1, Virchowweg 6, 10117 Berlin, Germany; 4, Neurocure Center for Excellence Charité - Universitätsmedizin Berlin; 5, Emory University, Atlanta, GA, USA.

Correspondence to: Robert.Sachdev@charite.de, Matthew.Larkum@charite.de

Number of pages: 43

Number of figures: 8

Number of extended figures: 6

Number of extended tables: 11

Words in abstract: 247

Words in introduction: 660

Words in discussion: 1518

Conflict of interests: The authors declare no competing financial interests.

Acknowledgements: We would like to thank: The Viral Core Facility of the Charité Universitätsmedizin Berlin for their support and the generation of the viruses used in this study; the AMBIO Facility of the Charité - Universitätsmedizin Berlin for their support; Kristin Lehmann for excellent technical assistance; Albert Gidon for input on an earlier version of the manuscript; Benjamin Judkewitz for providing the Drd1a-Cre and Ctgf-dgCre lines; Ehud Ahissar for the Gpr26-Cre line; Charles Gerfen for Tlx3-Cre and Sim1-Cre lines; James Poulet 
for importing the Gpr26-Cre and sharing the Vip-IRES-Cre line; and Edward Callaway for the following generous gifts: pAAV-EF1a-DIOHTB, pAAV-Ef1a-DIO-H2B-GFP-2A-oGWPRE-hGH, cDNA B7GG, BHKEnvA cells, and HEK293T-TVA cells.

Funding: European Union's Horizon 2020 research and innovation program and Euratom research and training program 20142018 (under grant agreement No. 670118 to MEL); Deutsche Forschungsgemeinschaft (Exc 257 NeuroCure, Grant No. LA 3442/3-1 \& Grant No. LA, Project number 327654276 SFB1315); European Union Horizon 2020 Research and Innovation Program (72070/HBP SGA1, 785907/HBP SGA2, 785907/HBP SGA3 670118/ERC Active Cortex); Einstein Foundation Berlin (EVF-2017-363), and NINDS (R01NS1114702).

Author contributions: J.L., T.A.Z., R.N.S., and M.E.L. planned and designed the experiments and wrote the manuscript. J.L., T.A.Z., and R.N.S. performed the experiments. J.L., T.A.Z., R.N.S., and M.T. analyzed the data. T.T., and C.R. produced viruses, designed experiments and wrote the manuscript. B.J.E. and D.J. wrote the manuscript. All authors approved the final version of the manuscript. 


\begin{abstract}
Neocortical layer (L) 1 is a locus for interactions between long-range inputs, L1 interneurons and apical tuft dendrites of pyramidal neurons. Even though we have a wealth of information about L1, the level and effect of local input to this layer have not been quantified. Here we characterized the input to L1 of mouse somatosensory cortex with fast blue, monosynaptic rabies and optogenetics. Our work shows that most of the input to L1 is local, and that both local and long-range inputs to this layer arise predominantly from L2/3 and L5 neurons. Subtypes of L5 and L6b neurons project to the overlying L1 with different probabilities. VIP and SST interneurons in L2/3 and L5 also innervate L1. A subset of local L5, the intratelencephalic, pyramidal neurons, drive L1 interneurons but have no effect on L5 apical tuft dendrites. Monosynaptic rabies-based retrograde labelling reveals presynaptic boutons covering the entire somato-dendritic axis of pyramidal neurons, including in L1. When fast blue application was combined with rabies virus, we found that only a fraction of local and long-range neurons was both presynaptic to L5 neurons and projected to L1. These results demonstrate that $\mathrm{L} 1$ receives a large proportion of its input from local neurons, and that some of these inputs specifically target interneurons. We conclude that L1 is not just a site for interaction between long-range feedback and apical tuft dendrites of pyramidal cells, it is also a site for complex modulation of pyramidal neurons and interneurons by local inputs.
\end{abstract}




\section{Significance Statement}

Here we used traditional retrograde tracing combined with physiology and rabies virus to quantify the connectivity to L1 of somatosensory cortex. We show that local inputs far outweigh long-range inputs. We also show that subtypes of L5 neurons target the overlying L1 and depolarize L1 interneurons but have little effect on the apical tuft dendrites of L5 neurons. Local and long-range cortical inputs to L1 arise from presynaptic neurons in L2/3 and L5. The long-range input arises from a variety of cortical areas including primary motor, secondary somatosensory and motor, perirhinal and visual cortex. Thus, interneurons in L1 and the dendrites of pyramidal neurons are the site of complex interactions with local and long-range inputs. 


\section{Introduction}

Neocortical layer (L) 1, long an enigma (Burkhalter, 1989; Gămănuţ et al., 2018; Hubel, 1982; Ibrahim et al., 2020; Marín-Padilla, 1998), has recently become central to ideas about consciousness and perception (Larkum, 2013; Aru et al., 2020; Suzuki and Larkum, 2020; Takahashi et al., 2016, 2020). L1 contains apical tuft dendrites that originate from somata of pyramidal neurons in L2/3 and L5. Decoupling the pyramidal neuron somata from their apical tuft dendrites in L1 has been related to unconscious states (Suzuki and Larkum, 2020) and activity in apical tuft dendrites has been associated with perception (Takahashi et al., 2016, 2020). These experiments support a hypothesis that links sensory data to "contextual” or "internal" data that interacts at the level of the pyramidal neuron and its dendrites (Aru et al., 2020; Larkum, 2013; Larkum et al., 2018; Shepherd, 2011).

What makes L1 special in this context? One influential line of thinking (Felleman and Van Essen, 1991) is that primary sensory cortical areas generate feedforward input to higher order cortical areas, which in turn provide feedback to lower order cortical areas. These ideas were expanded into a hypothesis with the pyramidal neuron and its apical tuft dendrites as key loci for the interaction between feedforward and feedback input (Larkum, 2013). When a pyramidal neuron receives strong feedforward input targeting its somatic region, action potentials are triggered that backpropagate into the apical dendrite. The hypothesis suggests that feedback inputs simultaneously activate the apical tufts (Takahashi et al., 2020), which leads to long-lasting calcium potentials in the apical dendrite and consequently bursts of action potentials (Doron et al., 2020).

L1 is a key locus for these interactions because here long-range cortico-cortical feedback can interact with the apical-tuft dendrites of pyramidal neurons. L1 is special in many regards: It is cell sparse (Ramon y Cajal, 1899a, 1899b in DeFelipe and Jones, 1988; Gabbott and Somogyi, 1986; Ibrahim et al., 2020; Lee et al., 2010; Marín-Padilla, 1998), but filled with axons from a variety of sources and dendrites of a variety of local neurons (Cauller, 1995; DeFelipe and Fariñas, 1992; Larkum, 2013; Schuman et al., 2021 Cajal, 1899a, 1899b in DeFelipe and Jones, 1988). It contains exclusively GABAergic inhibitory interneurons and apical tuft dendrites of pyramidal cells (DeFelipe, 1997; Ito et al., 1998; Karimi et al., 2020; Lee et al., 2010; Mason and Larkman, 1990; Rudy et al., 2011; Schuman et al., 2019). It consists of two sublayers as some inputs specifically target just the inner or just the outer portion of the layer (Cruikshank et al., 2012; Doron et al., 2020).

Earlier work with retrograde tracers fast blue and diamidino yellow has shown that long-range cortico-cortical projections are an important source of input to L1 (Cauller, 1995; 
Cauller et al., 1998). Reconstruction of motor cortical and thalamocortical axons has further seeded the idea that long-range inputs target L1 (Binzegger et al., 2004; Ohno et al., 2012; Rubio-Garrido et al., 2009; Veinante and Deschênes, 2003). Quantification of axons and boutons in L1 suggest a key role for the cortico-cortical input to this layer (Binzegger et al., 2004; Boucsein and Martin, 2011; Douglas and Martin, 2007). More recently, however work with rabies virus tracing has shown that while L1 NDNF positive interneurons receive longrange input, a large portion of their input is from local cortical neurons (Abs et al., 2018; CohenKashi Malina et al., 2021). Single-cell fills show that axons of L2/3 and L5 neurons arborize in L1 (Brown and Hestrin, 2009; Feldmeyer et al., 2006; Sakmann, 2017; Schuman et al., 2021). Paired recordings from L1 interneurons and L2/3 pyramidal cells show that L2/3 neurons can modulate the activity of L1 interneurons (Wozny and Williams, 2011).

Here we combined fast blue retrograde and rabies virus tracing with channelrhodopsin based circuit mapping to quantify the local and long-range input to L1 of primary somatosensory cortex. Our work shows that most input to L1 is from local neurons and that local L5 inputs specifically drive activity in L1 interneurons. 


\section{Methods}

Ethics statement. All experiments were conducted under the license G0278/16 in accordance with the guidelines of animal welfare of the Charité Universitätsmedizin Berlin and the local authorities, the 'Landesamt für Gesundheit und Soziales'.

Mice. We used adult wildtype C57BL6/J mice and transgenic Ai9 reporter mouse lines expressing layer-specific genes under the tdTomato (tdTom) promoter (Breeding Unit Steglitz, Charité Universitätsmedizin, Berlin): Tlx3-Cre for L5 intracortical (IT) projections (Tlx3-Cre Tg(Tlx3-cre) PL56Gsat/Mmucd (NIMH) MMRRC Stock 041158-UCD, lfd nr. 1287, Gerfen et al., 2013); Sim1-Cre for L5 pyramidal tract (PT) projections (Sim1-Cre Tg(Sim1-cre) KJ18Gsat/Mmucd, (NIMH) MMRRC Stock 031742-UCD, lfd nr. 1288, Gerfen et al., 2013), Drd1a-Cre and Ctgf-2A-dgCre for L6b neuronal subpopulations (Drd1a-Cre Tg(Drd1-cre) FK164Gsat/Mmucd, MMRRC Stock 030781-UCD, lfd nr. 1286, Benjamin Judkewitz, Charité; Ctgf-2A-dgCre Cg-Ccn2 < tm1.1 (folA/cre) Hze > /J, JAX Stock 028535, Benjamin Judkewitz, Charité), Sst-IRES-Cre for somatostatin positive interneurons (SST-IRES-Cre Sst $<$ tm2.1(cre)Zjh>/J, JAX Stock 013044), Vip-IRES-Cre for vasoactive intestinal peptide (VIPIRES-Cre Vip <tm1(cre)Zjh>/J, JAX Stock 010908), Gpr26-Cre Tg (Gpr26-cre), lfd nr. 1333, KO250Gsat/Mmucd; MMRRC Stock 033032-UCD (Ehud Ahissar, Weizmann Institute) for neurons of the posteromedial complex of thalamus (POm). We crossed the Cre-lines with Ai9 reporter mice to induce tdTomato expression in Tlx3, Sim1, Drd1, Ctgf, Vip and Sst neurons. The Cre expression in Ctgf-Cre mice was stabilized by intraperitoneal administration of trimethoprim ( $250 \mu \mathrm{g} / \mathrm{g}$ body weight). All mice were kept in groups of two to three individuals under standard conditions in a 12-hour day-night cycle at $21^{\circ} \mathrm{C}$ room temperature. Water and food were available ad libitum.

Rabies virus production. All viruses for rabies tracing were produced in the Viral Core Facility of the Charité, Universitätsmedizin Berlin (vcf.charite.de). The production was previously described by the Callaway lab (Osakada and Callaway, 2013). In brief, we transfected B7GG cells by Lipofectamine 3000 (Thermo Fisher) with the rabies virus genomic vectors that contained either mCherry (pSADdeltaG-F3-mcherry, Addgene plasmid \#32634), or GFP and Synaptophysin-RFP cDNA (pRVdG-N-P-M-EGFP-SynPhRFP-L, Addgene plasmid \#52483), and with these additional plasmids: pcDNA-SADB19N Addgene plasmid \#32630, pcDNA-SADB19P Addgene plasmid \#32631, pcDNA-SADB19L Addgene plasmid \#32632, pcDNA-SADB19G Addgene plasmid \# 32633. We collected the supernatant over several days and re-transfected the recovered virus in B7GG cells for a final collection step. For additional pseudotyping, the rabies with the envelope protein EnvA of the Avian Sarcoma 
and Leukosis virus (ASLV), and the obtained supernatant containing unpseudotyped viruses was applied onto BHK-EnvA cells. After three to five days, EnvA-pseudotyped rabies viruses containing supernatant were collected, filtered and concentrated by ultracentrifugation. Rabies virus titer was determined by infecting a serial dilution of the virus in HEK293T-TVA cells. All components for rabies virus production were gifts from Edward Callaway (Salk Institute, San Diego, CA, USA).

In order to allow expression in Cre positive starter cells, we applied a previously described AAV vector that contained a Cre dependent expression cassette of a nuclear GFP, EnvA interacting TVA receptor and an optimized rabies G protein (pAAV-Syn-Flex-nGToGWPRE3) (Choi and Callaway, 2011; Kim et al., 2016; Sun et al., 2014; Zolnik et al., 2020). To distinguish between rabies GFP expression and GFP expression from AAV infected starter cells, we exchanged the nuclear GFP with mCerulean3 (addgene plasmid \#54730) (Markwardt et al., 2011) to form pAAV-Syn-Flex-Ce3ToG-WPRE3. After sequence verification, AAV was produced at the Viral Core Facility of the Charité - Universitätsmedizin Berlin.

Preparation of mice for surgeries. We deeply anaesthetized the mice $(n=40)$ according to their weight with a mixture of ketamine $(100 \mathrm{mg} / \mathrm{kg})$ and xylazine (10 mg/kg). After full anaesthesia, confirmed by toe pinch for absence of reflexes, mice were placed into a stereotaxic frame with non-puncture ear bars and a nose clamp (Kopf Stereotaxic device, California, USA, Inc.). For analgesics, we applied $100 \mu \mathrm{l}$ of lidocaine under the scalp before surgery and administered Carprofen (5 mg/kg) and Buprenorphine (5 mg/kg) intraperitoneal for analgesic post-treatment after surgery. Body temperature was maintained at $37^{\circ} \mathrm{C}$, using a heating pad during the surgery and while mice recovered from the operation.

Fast blue application. Fast blue (fb) was applied on L1 of S1 cortex, where it is taken up for retrograde transport along the axons (Keizer et al., 1983; Kuypers et al., 1980). We made a craniotomy of $\sim 0.5 \mathrm{~mm}^{2}$ over S1 cortex (AP: -1.2 to $-1.7 \mathrm{~mm}$, lateral: $2.3 \mathrm{~mm}$ to $2.7 \mathrm{~mm}$ ) and without removing the dura mater applied $2 \mu \mathrm{l}\left(1 \%\right.$ in $\left.\mathrm{H}_{2} \mathrm{O}\right)$ of the retrograde, blue fluorescent neuronal tracer fast blue (Polyscience Inc, 17740-1, 1mg) onto the surface of L1 for 5 minutes. The fluid that remained on the brain was washed off with PBS. After the procedure, we cleaned the skull, sutured the scalp and put the mouse back into its home cage for recovery. Seven days later, brains were removed and processed for analysis as described below.

Definition of application site. For quantification of $\mathrm{fb}$, we defined the center of the application site by eye (as the slice with the most fb labelling). Note that fast blue could also spread from the application site at barrel cortex into lateral hindlimb / trunk S1 cortex, and that 
at the application site sections could be distorted. For analysis we used every second brain section and stored alternate sections for additional examination.

Stereotaxic viral and rabies injections. For stereotaxic injection, an incision was made in the scalp, the skull was cleaned with PBS, and a small craniotomy was drilled above the injection location using a dental drill. The brain tissue was kept moist by applying sterile PBS at regular intervals. For injections, we tip-filled pipettes using negative pressure, and injected with pipettes of a tip size of approximately $4 \mu \mathrm{m}$. Viral injections -- $200 \mathrm{nl}$ under constant positive pressure with a flow rate of $20 \mathrm{nl} / \mathrm{min}$-- were made using the following stereotaxic coordinates for S1 cortex: AP -1.5 mm, lateral: $2.7 \mathrm{~mm}$, ventral: $0.8 \mathrm{~mm}$; for POm: AP: -1.5 $\mathrm{mm}$, lateral: $1.5 \mathrm{~mm}$, ventral: $3.0 \mathrm{~mm}$.

To induce Cre specific expression of channelrhodopsin, an AAV1-Flex-ChR2-eYFP (1 x 10^13 vg/mL) was injected into S1 of Tlx3-Cre and Sim1-Cre mice. To induce rabies virus expression, we first injected $200 \mathrm{nl}$ AAV-Syn-Flex-nGToG-WPRE3 (8.1 x 1011 GC/mL) into L5a and L5b and allowed two to three weeks for expression, followed by injection of $200 \mathrm{nl}$ of RABVSADB19dG-mCherry (1.4 x $108 \mathrm{IU} / \mathrm{mL})$. When combining rabies virus injection with $\mathrm{fb}$ application, we applied $\mathrm{fb}$ as on the day of rabies virus injection and waited seven days for expression. We analyzed every second section. In rabies virus injections, GFP positive neurons were distributed from $600 \mu \mathrm{m}$ to $1100 \mu \mathrm{m}$ across the injection site.

Slice preparation for electrophysiology. We prepared coronal slices $(300 \mu \mathrm{m})$ from Tlx3-Cre and Sim1-Cre mice. Immediately following the slice preparation, we used ice-cold solution for slicing and incubation of the brain slices for $5 \mathrm{~min}$ at $32{ }^{\circ} \mathrm{C}$ in $(\mathrm{mM})$ : 110 choline chloride, $2.5 \mathrm{KCl}, 1.25 \mathrm{NaH}_{2} \mathrm{PO}_{4}, 26 \mathrm{NaHCO}_{3}, 11.6$ sodium ascorbate, 3.1 sodium pyruvate, $7 \mathrm{MgCl}_{2}, 0.5 \mathrm{CaCl}_{2}$ and 10 d-glucose, $\mathrm{pH} 7.4$, saturation $95 \% \mathrm{O}_{2} / 5 \% \mathrm{CO}_{2}$, then the slices were incubated for a further 25 minutes at $32{ }^{\circ} \mathrm{C}$ in normal artificial cerebrospinal fluid (ACSF, in mM: $125 \mathrm{NaCl}, 2.5 \mathrm{KCl}, 1.25 \mathrm{NaH}_{2} \mathrm{PO}_{4}, 25 \mathrm{NaHCO}_{3}, 2 \mathrm{CaCl}_{2}, 1 \mathrm{MgCl}_{2}$, and 25 dextrose, saturation $95 \% \mathrm{O}_{2} / 5 \% \mathrm{CO}_{2}$ ). ACSF was used for long-term incubation at room temperature and recordings in a submersion chamber at $32{ }^{\circ} \mathrm{C}$.

Definition of cell types for recording. We recorded from eYFP-negative neurons in L5 at the site of the viral injection (where expression was maximal). We classified neurons as L5b pyramidal cells if they had a single apical dendrite that extended to L1, their somata were about $800 \mu \mathrm{m}$ from pia, and they had electrophysiological characteristics of pyramidal tract neurons, such as large sag current upon hyperpolarization and low input resistance. L5a pyramidal neurons had somata less than about $700 \mu \mathrm{m}$ from pia, and a thin apical dendrite and small tuft in L1. Neurons with somata in L1 with non-spiny dendrites were classified as L1 interneurons. 
Recording and imaging. Whole-cell current clamp recordings with low resistance (4-7 $\mathrm{M} \Omega$ ) microelectrodes were made using a Dagan (Minneapolis, MN) BVC-700A amplifier. For current-clamp recordings the intracellular solution contained (in mM): $115 \mathrm{~K}$-gluconate, $5 \mathrm{KCl}$, 10 HEPES, 4 ATP-Mg, 0.3 GTP-Tris, and 10 phosphocreatine, 0.1\% biocytin. The pH was adjusted with $\mathrm{KOH}$ to 7.25-7.30 (285-295 mOsm). The liquid junction potential was not corrected. Slices were visualized on an Olympus (Tokyo, Japan) BX50WI microscope using a Photometrics CoolSnap ES (Tuscan, AZ) CCD camera and oblique optics. During recording or post-recording, neurons were filled, identified and categorized using Alexa Fluor 594 fluorescent dye (Invitrogen, Carlsbad, CA; $10 \mathrm{mM}$ ) and/or biocytin (0.2\%).

Channelrhodopsin based circuit mapping was used to test the effect of Tlx3-Cre or Sim1Cre input to L1 interneurons and to examine connections between L5 pyramidal neurons. We injected AAV1-Flex-ChR2-eYFP (1 x 10^13 vg/mL) into S1 of Tlx3-Cre and Sim1-Cre mice. Following three to five weeks of expression, we sliced the brains and recorded from neurons in L1 and L5 in the region of the densest ChR2-eYFP label. ChR2 photocurrents and EPSPs were distinguishable based on the response time during optical stimulation. Recordings of synaptic input were done in the presence of tetrodotoxin (TTX) and 4-aminopyradine (4-AP) to ensure that the connections being activated were monosynaptic.

Optogenetics and stimulation. An X-cite 200W mercury lamp was used to target optogenetic stimulation to L1 (Excelitas Technologies, Mississauga, Ontario, Canada) through a light guide gated by a millisecond shutter. Light was then filtered with a 475/30 nm bandpass filter and delivered to the slice through the epifluorescence port. A LUMPlanFl 60x/0.90 W Olympus immersion objective was used during these experiments. The power output at the objective was $>5 \mathrm{~W} / \mathrm{cm}^{2}$, which was about 100 -fold above activation threshold for channelrhodopsin. Light pulses were $10 \mathrm{~ms}$ in duration.

Pharmacology. The recordings were done in the presence of TTX $(0.5 \mu \mathrm{M})$ and 4-AP (1.2 $\mathrm{mM})$ following baseline recordings.

Histology and Nissl stain. Mice were deeply anesthetized with isoflurane (Baxter Inc.), and transcardially perfused with $0.1 \%$ PBS for three minutes, and subsequently with $4 \%$ paraformaldehyde (PFA) in 0.1M phosphate buffer (PB, $\mathrm{pH} 7.2$ ) for seven minutes. Brains were carefully removed from the skull and post-fixed in $4 \%$ PFA overnight at $4{ }^{\circ} \mathrm{C}$. For sectioning with a vibratome, brains were embedded in $3 \%$ agarose, for sectioning with a microtome brains were soaked in 30\% sucrose overnight. All brains were sectioned at $70 \mu \mathrm{m}$ to $100 \mu \mathrm{m}$. Every second section was mounted on slides and cover slipped. The remaining sections were stored in cryo-protect medium (30\% Ethylene Glycol, 30\% Glycerol in PBS) at 
$-20{ }^{\circ} \mathrm{C}$. We performed nissl (nissl) stain with NeuroTrace ${ }^{\mathrm{TM}}$ 500/525 Fluorescent Nissl Stain (Thermofisher, N21480) according to the protocol: Brain slices were washed in PBS three times, and then incubated in nissl solution (1:500) for $20 \mathrm{~min}$. After the labelling step, brain sections were washed three times with PBS and mounted in glycerol (80\%, 2.5\% DAPCO in PBS).

Confocal imaging. Images were taken on confocal laser scanning microscopes (Leica SP5, Leica SP8, Nikon A1Rsi+), or a widefield microscope (Nikon Widefield Ti2). Images were obtained with a 10x air, and 20x-, 40x-, or 63x oil immersion objectives (Leica: HCX PL APO 20x/0.7, HCX PL APO 63x/1.20 W motCORR CS; Nikon 20x Plan Apo, Air, 0.8 NA, 1.000 DIC N2 VC, Nikon 40x Plan Flour, Oil, 1.3 NA, 200WD, DIC N2 H; Nikon 10x Plan Fluo Air, 0.3 NA, 15.200 WD, Ph 1 DL). Fast blue was imaged with a 405 nm, GFP with a 488 nm, and tdTomato/ mCherry/ RFP with a 561 nm laser (bandwidth, Leica, 425/70, 515/25, 590/70; Nikon, 450/50, 525/50, 595/50; Nikon widefield, 435/33, 519/26, 595/31).

Cell counting and definition of cortical areas and cortical layers. Cell bodies were manually counted using the cell counter plugin from Fiji/ ImageJ. Cortical areas were defined according to the Allen brain reference atlas (https://mouse.brain-map.org/) and the Paxinos Atlas (Franklin, K. B. J. and G. Paxinos, 2008, The mouse brain in stereotaxic coordinates, Academic Press / Elsevier). The coordinates we used for each area were: M1 (AP 1.0 to 1.5, lateral 1.0 to 1.5), M2 (AP 2.0 to 2.5, lateral 1.0 to 1.5), S1 including trunk/ limb/ barrel field (AP -1.0 to -1.8 , lateral 2.3 to 2.7), S2 (AP -1.0 to -1.8 , lateral 2.7 to 3.5 ), visual cortex, including V1, V2 (AP 2.9 to 4.0, lateral 1.5 to 3.0), perirhinal cortex (AP -1.5 to -3.5, lateral 3.0 to 3.8).

Cortical layers in the brain areas were identified according to the Allen brain reference atlas and in bins of 100/ 200 microns for each layer. In S1 cortex were identified according to

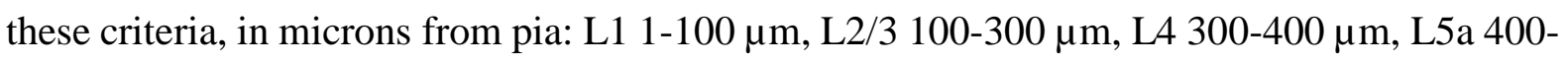
$500 \mu \mathrm{m}, \mathrm{L} 5 \mathrm{~b}$ 500-700 $\mu \mathrm{m}, \mathrm{L} 6 \mathrm{a}, 700-900 \mu \mathrm{m}$. Layers for M1 and M2 cortex were identified according to these criteria, in microns from pia: L1, 1-100 $\mu \mathrm{m}, \mathrm{L} 2 / 3,100-300 \mu \mathrm{m}, \mathrm{L} 5 \mathrm{a}, 300-$ $400 \mu \mathrm{m}$, L5b, 400-600 $\mu \mathrm{m}$, L6a, 600-800 $\mu \mathrm{m}$. For S2, V1 and V2, we set the criteria for layers

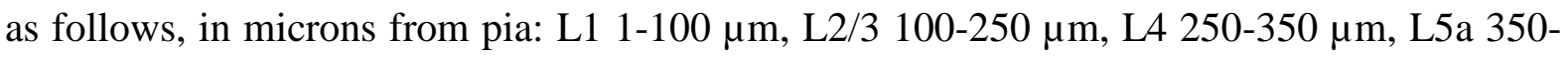
$450 \mu \mathrm{m}$, L5b 450-600 $\mu \mathrm{m}$, L6a 600-800 $\mu \mathrm{m}$. In all these areas, L6b was defined as the cell layer within 100 microns above the white matter. Note that in our scheme L5a was 100 microns and L5b was 200 microns. These definitions are to some extent arbitrary (Oberlaender et al., 2011). To examine how this definition of layers affects our results, in a subset of brains, we also counted labeled neurons in a 30-micron strip in the middle of each 100-micron bin. 
Statistics. Statistical analyses were carried out with graphpad/ Prism. Data are shown as mean \pm S.E.M. (standard error of the mean). p-values are indicated in the text: ${ }^{*} \mathrm{p}=0.05-0.01$, ${ }^{* *} \mathrm{p}=0.01-0.001,{ }^{* * *} \mathrm{p}=0.001-0.0001 ;{ }^{* * * *} \mathrm{p}=<0.0001$. For figures $1,2,3,4,6,7$, and 8 , a oneway ANOVA, Bonferroni post hoc test was used, for figure 5, a Fisher’s Exact test was used. 


\section{Results}

\section{Input to $L 1$ assessed by fb application}

Local input to L1. Seven days after application of the retrograde tracer fast blue (fb) on L1 in somatosensory (S1) cortex (Figure 1A), fb labeled neurons were evident at the application site and in sites distributed throughout the brain. We first examined the proportion of all neurons that had taken up fb under the application site. We found the highest uptake of $\mathrm{fb}$ in L2/3 (42\% of all labeled neurons under the application site) and in L5 (L5a: 18\%, L5b: 11\%). L4 and L6a had fewer fb labeled neurons (L4, 5\%, L6a, 1\%), and L6b had 10\% of fb labelled neurons. The proportion of fb of total in L1 was $12 \%(n=6,3096 \pm 459$ neurons per brain, statistical analysis with one-way ANOVA $* * * * p<0.0001$; Figure 1B, Table 1-1). These distributions are in broad agreement with the earlier qualitative observations in rats (Cauller et al., 1998; Clancy and Cauller, 1999).

Laminar pattern of local input to L1. Next, to determine the percentage of neurons in each layer that project to L1 under the application site, sections were stained with nissl and the number of double labeled (nissl and fb) neurons for each layer were counted. This approach shows that most neurons in L2/3 (51\%) and in L5 (L5a, 35\%, L5b, 34\%) were double labeled Fewer neurons in L4 (26\%) and L6a (5\%), and 36\% of L6b neurons were double labeled. Finally, $46 \%$ of neurons in L1 were double labeled (statistical analysis with one-way ANOVA ${ }^{* *} \mathrm{p}<0.01$; Figure 1C, Table 1-1). These results suggest that the majority of L2/3 and L5 neurons project to L1.

Input to S1 L1 from contralateral S1. Contralateral to the application site, most fb labeled neurons were found in L2/3 (61\% of all labeled neurons) and fewer were found in L5 (L5a: 25\%, L5b: 12\%). Very few neurons in L4 (1.7\%) and L6a (0.3\%) were labelled. No neurons in L1 and L6b were labelled. Note that the total number of fb labeled neurons in the contralateral S1 (366 \pm 55 neurons per brain) were $10 \%$ of the total number of fb labeled (3096) neurons in the ipsilateral S1 (statistical analysis with one-way ANOVA **** $\mathrm{p}<0.0001$; Figure 1D, Table 1-1).

Laminar pattern of input to S1 L1 from contralateral S1. Nine percent of L2/3 neurons (fb and nissl stained) in contralateral S1 project to S1 L1 in the other hemisphere. In L5a 4\%, and in L5b 3\% were fb labelled. No neurons were double labelled in L1, L4, L6a, or L6b (Figure 1E, Table 1-1).

Local versus long-range input to L1. When this approach of counting fb labeled neurons, was extended to the whole brain, we find that that the local input to L1 of S1 arises predominantly from neurons underlying the application site (76\%), with long-range input 
constituting $24 \%$ of the total input (n=4, 11956 \pm 2469 neurons per brain, Figure 1F, Table 12). The long-range input to L1 was from higher order thalamus (7\%), ipsilateral motor cortices (9\%), S2 cortex (2\%), visual cortices (3\%), and perirhinal cortex ( $0.1 \%$ ipsi and $0.4 \%$ contra). Contralateral S1, motor, and perirhinal cortex also contributed input to L1 of somatosensory cortex. Note that we did not quantify input from brainstem to L1.

Laminar pattern of long-range input to L1 from other cortical areas. To obtain an estimate of laminar distribution of input to S1 cortex, we assessed fb labelled neurons in other cortical areas (Figure 2, Table 2-1). In higher order motor cortices (M2), most fb labeled neurons were in L2/3 and L5a, with a significantly larger number of fb labeled neurons in L5a (35\%) than in L2/3 (14\%) (statistical analysis with one-way ANOVA **** $\mathrm{p}<0.0001$; Figure 2A). In M1, most fb labeled neurons were distributed roughly equally in L2/3 (19\%) and L5a (21\%) (Figure 2B). In S2 cortex, most fb labeled neurons were located in L2/3 (42\%). Other layers had a smaller number of fb labeled neurons (Figure 2C). In visual cortices, most fb labeled neurons were located in L2/3 (42\%) followed by L5a (23\%) (Figure 2D). In perirhinal cortices, neurons in L5a from both hemispheres project to S1 L1 (Doron et al., 2020), but the bulk of the perirhinal projection to L1 of S1 were from the contralateral hemisphere. The perirhinal input arose from neurons in L5a (Figure 2E), which accounted for $2 \%$ of neurons in L5a in ipsilateral and 22\% in contralateral perirhinal cortex (fb/nissl, data not shown). Fast blue labeled neurons were also found in subcortical areas, POm, zona incerta, midline thalamic nuclei, basal forebrain and brainstem. Note that subcortically we only counted the number of $\mathrm{fb}$ labeled neurons in the thalamus and zona incerta (Figure 2F), we made no attempt to determine the percentage of labeled neurons (i.e. the ratio of fb labeled neurons to nissl stained cells).

Methodological considerations for the application of $\mathrm{fb}$ on $\mathrm{S} 1 \mathrm{~L}$. A first issue that arises with the application of fb on the surface of cortex is that there is no method for controlling fb spread, it can easily spread into L2/3 or even L4. To address this issue, we examined whether VPm/ VPl thalamus had fb labeled neurons and excluded brains with label in VPm/ VPl (Figure 1-1A).

A second issue that arises is that label at the site of application can be dense and it can be difficult to separate labeled neurons from background. To address this issue, we also counted $>140$ microns off the application site. When this approach was applied, labelling in S1 cortex had a similar laminar pattern (n=6, $1645 \pm 379$ neurons per brain, Figure 1-1B, Table 1-1) but at $>140$ microns from the application site there was an $8 \%$ reduction in the percentage of local fb labeled neurons ( $n=4,8176 \pm 2150$ neurons per brain, Figure 1-1C, Table 1-2). We then used 
another approach to examine this issue and counted in L5 and L6 where there is little background label from fb application. In L5 and L6, the counts under the application site, and at $>140$ microns from the application site, were slightly but significantly reduced, but the overall pattern of label in L5 and L6 was similar at both sites (Figure 1-1D). Finally, we counted in L6b, laterally away from the application site. When we do this there was a steady decrease in number of labeled neurons for L6b (Figure 1-1E). Taken together, this suggests that the laminar pattern of fb label was similar whether counts were made at the center of the application site or >140 microns from the center.

A third issue that arose was the delineation of layers at the application site (Figure 1C). A general consideration when defining layers is that brain sections are not uniform, they curve and can shrink differentially from animal to animal. This affects the definition of layers and makes it somewhat arbitrary - in particular for the definition of layers 2 through layer 6a. L1 and L6b on the other hand are easily defined by their location and histological appearance. In our dataset this issue becomes important for distinguishing the borders between L2/3, L4 and L5 under the application site where fb label is dense and the tissue can be deformed. Therefore, in an additional analysis we restricted our counts to 30-micron strips in the middle of each 100micron bin used to delineate each layer (see methods). When we do this, we find that the proportion of fb labeled neurons (fb and nissl) was reduced in all layers: $45 \%$ of the L2/3 and $53 \%$ of the L5 were fb labeled. The percentage of fb labeled neurons in L4 dropped to $4 \%$, and to $0.4 \%$ in L6a. In L6b, 32\% of neurons were fb labeled, and in L1 43\% fb labeled (Figure 11F, Table 1-2, one-way ANOVA **** $\mathrm{p}<0.0001$ ). These results suggest that some of the labeled neurons that were classified as being in L4 could be on the border of L2/3 and L5. Because the laminar pattern of label is similar with both approaches, we used 100-micron bins as the general approach to delineate each layer (see methods for additional details).

A final issue that arises with application of fb on the surface of the cortex is that it is not clear whether the standard method of injecting tracers deeper into cortex would produce a different pattern of retrogradely labeled neurons. To address this, we examined the effect of injecting fb into S1 cortex (Figure 2-1, Table 2-1-1). When fb was injected into cortex (Figure 2-1A), uptake of $\mathrm{fb}$ at the injection site was highest in $\mathrm{L} 2 / 3$ and evenly distributed in the other layers (one-way ANOVA $* * \mathrm{p}<0.01$; Figure 2-1B). In contralateral $\mathrm{S} 1$, fb uptake was highest in L2/3 and evenly distributed across the cortical layers (Figure 2-1C). In S2, the largest number of fb labeled neurons were found in L2/3 and L6a (one-way ANOVA, ${ }^{*} \mathrm{p}<0.05$; Figure 2-1D). In motor cortices, fb neurons were distributed across all layers (Figure 2-1E). In thalamic areas, in addition to labeled neurons in POm and zona incerta, neurons in VPm and 
VPl were also labeled (Figure 2-1F). These results suggest that long-range projections to L1 consist of a specific set of neurons located in L2/3 and L5 (and in some cases L6b) whereas long-range feedback to deeper layers of cortex engages an additional set of neurons in L6a.

\section{Cell type specific projections to S1 L1}

To assess the cell type specificity of input to L1, we examined fb uptake after application on the surface of L1 in somatosensory cortex in Ai9-reporter mice (Figures 3, 4, Tables 3-1, 4-1). We used Tlx3-Cre mice for L5 intratelencephalic neurons (IT) and Sim1-Cre mice for L5 pyramidal tract (PT) neurons (Gerfen et al., 2013; Gong et al., 2003). For subclasses of L6b projection neurons we used Ctgf-2A-dgCre and Drd1a-Cre mice (Heuer et al., 2003; Hoerder-Suabedissen et al., 2018; Zolnik et al., 2020). To examine the inhibitory component of input to L1, we used Vip-Cre for vasoactive intestinal peptide, and Sst-Cre for somatostatin neurons (Taniguchi et al., 2011).

Layer 5 IT and PT neurons in S1. Tlx3-Cre neurons were exclusively found in L5, primarily in L5a. They constitute $42 \%$ of nissl stained neurons in L5, with 76\% in L5a, and the remaining in L5b (Figure 3A). By comparison, Sim1-Cre neurons were distributed from L4 to L6, with 54\% in L5b, and 28\% in L5a and the rest in other layers. Overall, Sim1-Cre neurons constituted 18\% of all neurons in L5 (Figure 3B).

Local projections from IT and PT neurons to L1. To examine the IT and PT connectivity to L1, we applied fb on L1 in Tlx3-Cre-Ai9 and in Sim1-Cre-Ai9 mice and counted double labeled neurons in the two lines. In ipsilateral S1, 29\% of all IT neurons in L5 were fb labeled, with majority located in L5a (one-way ANOVA, **** $\mathrm{p}<0.0001$; Figure 3C, Figure 3-1A, Table 3-1-1). The PT neurons projecting to L1 showed a different profile, with $20 \%$ of all PT neurons double labeled for fb ipsilaterally, with the majority of L1 projecting neurons located in L5b (Figure 3D, Figure 3-1B, Table 3-1-1).

Long-range projections of PT and IT neurons to L1. IT and PT neurons in contralateral S1, ipsilateral motor cortices and S2 cortex were labeled with fb (Figure 3C, 3D). For contralateral S1, 8\% of IT neurons (Figure 3C), and only 1\% of PT neurons were labeled (Figure 3D). The percentage of double labeled neurons in motor cortices was similar for IT and PT neurons ( 10\%). In S2, 11\% of IT neurons, and 8\% of PT neurons were double labeled. Thus, IT neurons project contralaterally to L1 and slightly more IT than PT neurons in motor and S2 cortices have long-range projections to L1. Overall, 1.5\% of the IT neurons in perirhinal cortex project to contralateral S1 L1. 
Local projections from L6b to L1. To examine the distribution of projections from L6b to L1, we applied fb on S1 cortex in Ctgf-Cre-Ai9 and in Drd1a-Cre-Ai9 mice. All Ctgf neurons were located in L6b, but Ctgf neurons constituted only 15\% of all neurons in L6b (Figure 3E). By comparison, Drd1a positive neurons were distributed in L6a and L6b, with 76\% of all Drd1a neurons in L6b. Drd1a positive neurons were 43\% of all neurons L6b (Figure 3F).

A larger proportion, 52\%, of Ctgf neurons projected to L1, while only $13 \%$ of Drd1a neurons projected to L1 (Figure 3G, 3H). No Ctgf or Drd1a positive fb double labeled neurons were found in the contralateral hemisphere and even though some L6b neurons in M1 do project to L1 S1, no Ctgf or Drd1a neurons in M1 had projections to L1.

Inhibitory input to L1. To examine the distribution of inhibitory projections to L1, we applied fb on L1 in Vip-Cre-Ai9 and in Sst-Cre-Ai9 mice (Figure 4, Table 4-1). First, we counted the number of Vip and Sst positive neurons in S1 cortex and found that in the cortical column 13\% of neurons were Sst positive (3220 tdTom out of 23889 nissl), and 5\% (460 tdTom out of 9780 nissl) were Vip positive (Figure 4A, 4B). These neurons were distributed in all cortical layers.

Fast blue labeled Sst neurons were most abundant in L2/3 and L5a, and fb labeled Vip neurons were most abundant in L2/3 (Figure 4C, 4D). There were also a small number of long-range projecting Sst inhibitory neurons in contralateral S1, in L2/3 and L5a that targeted L1 (0.3\%, 15 out of 6173 neurons, 3 brains).

\section{Functional effect of intra-L5 monosynaptic connections in L1}

These anatomical results suggest that a substantial input to L1 arises from local L2/3 and L5 neurons. The synaptic connectivity between L2/3 neurons and neurons in L1 has in part been examined previously (Wozny and Williams, 2011), so here we focused on the effect of L5 inputs to L1. We examined the synaptic connectivity between L5 pyramidal neurons and L1 interneurons as well as between L5 pyramidal neurons themselves (via connections within L1). We patched L1 interneurons or L5 pyramidal neurons in Tlx3-Cre or Sim1-Cre mice expressing ChR2. To examine input to L1 interneurons, we photo-stimulated over L1 while recording L1 interneurons. To examine connectivity between L5 pyramidal neurons we photostimulated at different points along the somatodendritic axis of L5 pyramidal neurons in the presence of TTX and 4-AP. This approach blocks di-synaptic transmission (Petreanu et al., 2007). We expected the IT input to generate a larger PSP than the PT input, in part because more IT neurons project to L1. The PSP parameters -- amplitude, latency, and short-term plasticity (at $10 \mathrm{~Hz}$ ) -- evoked in interneurons and L5 pyramidal neurons were measured but 
due to the use of TTX and 4-AP, which can alter normal release characteristics, these were not characterized in depth.

Effect of L5 input on L1 interneurons. We recorded from interneurons in L1 in Tlx3-Cre or Sim1-Cre slices while photo-stimulating in L1 (Figure 5A). Photo-stimulation of the axons in L1 showed that IT neurons generate a powerful PSP in L1 interneurons and that this input was significantly stronger than the PT input to the interneurons (Figure 5B, 5C; $n=5$ neurons, Tlx3 to L1 interneuron amplitude: $25.5 \pm 4.9 \mathrm{mV}$; Sim1 to L1 interneuron amplitude: $0.9 \pm 0.2$ $\mathrm{mV},{ }^{* * * *} \mathrm{p}<0.0001$, Student t-test). Also, note that $5 / 5$ interneurons received input from IT while only 2/5 interneurons received input from PT. The input from IT to L1 interneurons had depressing synaptic dynamics at $10 \mathrm{~Hz}$ stimulation (Figure 5D).

IT-PT interactions with other L5 pyramidal neurons. Next, we examined whether IT neurons could drive non-Tlx3 neurons in L5b, and whether PT neurons could drive non-Sim1 neurons in L5a (Figure 5E, 5F top). In order to restrict the transmitter release to the photostimulated area, and to prevent di-synaptic activation, we applied TTX and 4-AP to the bath solution. The entire somato-dendritic axis of the filled neurons was systematically photostimulated by moving the optogenetic light in $50 \mu \mathrm{m}$ steps, while simultaneously measuring the amplitude of the evoked EPSP (Figure 5E, 5F, middle). Activation of the Tlx3-Cre axons did not evoke any EPSPs in L5b tufts in L1. Similarly, activation of Sim1-Cre axons did not evoke EPSPs in non-Sim-L5a tufts in L1 (Figure 5E, bottom graph). The Tlx3 neurons did, however, evoke a significant depolarization (while photo-stimulating) at the oblique dendrites and to a lesser extent the basal dendrites and soma in L5b $(14.97 \pm 1.56 \mathrm{mV})$. The Sim1-Cre axons on the other hand, had a negligible effect on non-Sim L5a neurons across the entire somato-dendritic axis (2.30 $\pm 0.46 \mathrm{mV}$ ) (Figure 5F, bottom graph) (Ramaswamy et al., 2012).

In summary we show that the L5 Tlx3 IT neurons strongly drive interneurons in L1 but have a negligible effect on the apical tuft dendrites in L1 of non-Tlx3 L5b neurons. Layer 5 Sim1 PT neurons do not drive interneurons in L1 or the tuft dendrites of L5 non-Sim1 neurons in L1. This suggests that two major classes of L5 pyramidal neurons do not interact with each other directly within L1.

\section{Rabies approach targeting the L5 PT and IT neurons combined with fb application}

The experiments so far revealed that neurons from a variety of sources project to L1. Earlier work has shown that in addition to targeting L1, long-range input from cortical areas like M1 targets infragranular layers (Kinnischtzke et al., 2016; Yamawaki et al., 2021; Zagha 
et al., 2013). These observations raise a question: Do neurons that project to L1 have an axon collateral in L5? Or another way to ask the question: Are neurons that project to L1 presynaptic to the L5 pyramidal neurons? To address this question, we combined fb application in L1 with rabies-based retrograde monosynaptic tracing for the IT and PT neurons. Monosynaptic rabies tracing labels neurons connected by one synapse to the source (starter) neurons (Kim et al., 2015, 2016; Luo et al., 2008; Wickersham et al., 2007).

Distribution of input along the somato-dendritic axis. To examine whether the rabies virus targets synapses along the entire soma-dendritic arbor of the starter neuron -- i.e. to examine whether boutons of presynaptic neurons could be distributed in L1 to L6 -- we used a modified rabies virus linked to synaptophysin (Wickersham et al., 2013). With this approach, presynaptic cell bodies and their axons / boutons were labeled with synaptophysin linked to RFP and the starter population was additionally labeled with cystolic GFP and nuclear cerulean fluorescent protein (CFP) (Figure 6-1A). We examined the distribution of boutons and presynaptic label in a circuit where it was known that a class of presynaptic neurons target specific dendritic loci. For this assay, we used the Gpr26-Cre line, which expresses Cre in a class of CA1 pyramidal neurons (Figure 6-1B). Entorhinal neurons target the apical tuft dendrites of CA1 pyramidal neurons (Masurkar et al., 2017), so finding presynaptic neurons in entorhinal cortex would indicate that the rabies strategy is effective even for inputs that target the tuft dendrites. When we examined entorhinal cortex, we found labeled presynaptic neurons, suggesting that rabies retrograde approach can work for synapses at the tuft dendrites (Figure 6-1C).

Next, we used the same approach in S1 cortex of Sim1-Cre and Tlx3-Cre mice (Figure 6-1D). Starter cells expressing CFP were found in L5, and GFP expressing cells and RFP positive boutons were detected in all layers, including L1, across the cortical column (Figure 6-1E; Tlx3-Cre, $\mathrm{n}=369$ synapses, Sim1-Cre, $\mathrm{n}=158$ synapses, bouton counts from 10 boxes for each layer with a size of $20 \mu \mathrm{m}$ x $10 \mu \mathrm{m}$ ). Presynaptic neurons were also detected in motor cortex, S2 and thalamus (Figure 6-1F). Our qualitative assessment suggests that the rabies approach targets the entire somato-dendritic compartment -- including synapses on apical tuft dendrites in L1 -- of the starter pyramidal neurons.

L1 projecting neurons, presynaptic to PT and IT neurons. With these results in hand, we performed injections in Tlx3-Cre and Sim1-Cre mice with the standard AAV-rabies virus approach in combination with fb application to see whether neurons that were presynaptic to L5 neurons also project to L1 (Figure 6A). In Tlx3-Cre mice, fb application in L1 labeled 9596 
neurons, mCherry was found in 6138 cortical neurons presynaptic to the Tlx3 neurons. There were 449 neurons that were double labeled, i.e. were presynaptic to Tlx3 neurons and projected to L1 (Figure 6B, Table 6-1). In Sim1-Cre mice, there were $13131 \mathrm{fb}$ labeled, 6495 presynaptic neurons, and 823 double labeled neurons, i.e. those that were presynaptic to Sim1-Cre neurons and projected to S1 L1 (Figure 6B, Table 6-2). Note that we obtained similar results for fb application on L1 in these two transgenic lines as we do in wildtype mice (Figure 6-2).

Local intra-cortical pattern. Total presynaptic input was 6138 neurons in Tlx3 mice and 6495 neurons in Sim1 mice. Local presynaptic input to the Tlx3 neurons constituted 80\% of all input; for Sim1 local input constituted 63\% of total input (Figure 6C, 6D, Figure 8A, 8B, Table 8). Example images for the injection site in Tlx3-Cre and Sim1-Cre brains show starter cells in yellow and mCherry+fb double labeled cells in cyan (Figure 6C, 6D). Locally, in Tlx3-Cre brains, the presynaptic labeled neurons were mainly seen in L2/3 and L5 (one-way ANOVA, ${ }^{* * *} \mathrm{p}>0.001$; Figure 6C). Neurons that were double labeled i.e, were presynaptic to Tlx3 neurons and labeled with fb were most common in L2/3 and L5a (Figure 6C). Similarly, in Sim1-Cre brains, most of the local presynaptic input was from L2/3 and L5b (Figure 6D) and double labeled neurons were detected mainly in L2/3 and L5b.

Long-range input to L1 and L5 neurons. Long-range presynaptic input to Tlx3-Cre and Sim1-Cre neurons in S1 was primarily from motor cortices, S2 cortex and thalamus (Figure 7). Here we focused our count of presynaptic neurons on the areas that provide input to L1. In both, Tlx3 and Sim1 brains, long-range input arose from neurons in L2/3 and L5 and double labeled neurons -- i.e., neurons that were presynaptic to L5 cells and projected to L1 of S1 -were most commonly found in L2/3 (one-way ANOVA, ***p>0.001).

In S2 of Tlx3-Cre brains, presynaptic input was from L2/3 and L5a neurons (with a smaller input from L6a and L6b) and 49 neurons were double labeled for fb and mCherry (Figure 7A). None of the L6 neurons were double labeled.

L2/3 and L5a neurons in motor cortex provided most of the presynaptic input to S1 Tlx3 neurons, and few were double labeled (Figure 7B). Some presynaptic neurons were found in L6a and none were double labeled.

In S2 of Sim1-Cre brains, L2/3 and L5b neurons generated the bulk of the presynaptic input and 31 neurons were double labeled (Figure 7C). Presynaptic neurons in motor cortex were found in L2/3, L5a and L5b, but only 13 were double labeled (Figure 7D). Presynaptic neurons to both L5 lines were found in thalamus (Figure 7E, 7F), with slightly more input to Tlx3 neurons than to Sim1 neurons. Double labeled neurons accounted for a small percentage of labeled thalamic neurons in both lines (Tlx3: 1.8\%, Sim1: 4.1\%, Figure 7E, 7F). 
A control for $f b$, when combined with rabies approach. To examine whether $\mathrm{fb}$ application on L1 interferes with rabies, we also applied the rabies approach to Tlx3-Cre and Sim1-Cre lines without fb application. The distribution of presynaptic input was similar in both cases (Figure 6-3, Table 6-3-1). In Tlx3-Cre brains, presynaptic neurons were mainly found in L2/3 and L5a, in Sim1-Cre brains presynaptic neurons were mainly found in L2/3 and L5b, results which are similar to those in Figure 6C and 6D.

Taken together these results suggest that the bulk of the presynaptic input to Tlx3 and Sim1 neurons was from local neurons (Tlx3: 80\%, Sim1: 63\%). A large portion of the input targeting L1 that was presynaptic to L5 neurons, arose from local neurons. A fraction of the neurons presynaptic to L5 pyramidal neurons also projected to L1. Long-range cortical input to these pyramidal cells was from S2, motor and visual cortices (Figure 8A, 8B, Table 8-1). 


\section{Discussion}

Layer 1 has been dubbed an enigma, a crowning mystery (Hubel, 1982; Rudy et al., 2011) with its anatomy, connectivity, and function largely uncharted. Here we begin to unravel a portion of this mystery. At the outset of our study, it was known that neurons in L2/3, L5 and L6b provided input to L1 (Cauller, 1995; Cauller et al., 1998), and local inputs were in position to activate L1 interneurons, in fact L2/3 neurons activated these interneurons (Brown and Hestrin, 2009; Cauller, 1995; Cauller et al., 1998; Narayanan et al., 2017; Oberlaender et al., 2012; Sakmann, 2017; Wozny and Williams, 2011; Zolnik et al., 2020). It was also known that long-range inputs from a variety of sources targeted L1 (Cauller, 1995; Cauller et al., 1998; Doron et al., 2020; Larkum, 2013; Mease et al., 2016; Oda et al., 2004; Veinante and Deschênes, 2003; Ohno et al., 2012; Zagha et al., 2013; Sakmann, 2017) and that the bulk of input to neurogliaform interneurons in L1 arose locally (Abs et al., 2018; Cohen-Kashi Malina et al., 2021).

Our characterization of anatomical and functional input to L1 adds to the growing knowledge and reveals key organizational principles for L1 of S1 cortex: 1) Local inputs to L1 numerically outweigh the long-range inputs; 2) local and long-range L2/3 and L5 neurons provide the bulk of input to L1; 3) $10 \%$ to $20 \%$ of the PT and IT neurons in ipsilateral S2 and M1 and contralateral S1 project to L1 of S1; 4) Vip and Sst neurons especially in L2/3 target L1; 5) Input to L1 from local L5 pyramidal neurons has specific functional consequences, it targets L1 interneurons but not the dendrites of L5 pyramidal neurons in L1; 6) S2 input to L5 PT and IT neurons constitutes more than $40 \%$ of the total long-range input to these neurons; 7 ) A small proportion of neurons that are presynaptic to PT and IT neurons also project to L1.

Input to L1 is primarily local and can modulate activity of L1 interneurons. It is widely accepted that the majority of synapses in any patch of cortex arise from cortical neurons with subcortical, thalamic inputs, contributing $~ 15 \%$ of all synapses in cortex, even in L4 (Douglas and Martin, 2004; Binzigger et al., 2004; White, 1989; Shepherd 2004, Chapter 12, Neocortex. Douglas, Markram and Martin). An earlier, detailed analysis of axons and boutons of 39 filled neurons including both excitatory and inhibitory neurons and all layers of cat visual cortex, suggested that non-local sources could provide a bulk of the input -- "dark synapses" -- to L1 (Aertsen et al., 2010; Binzegger et al., 2004; Boucsein et al., 2011; Douglas and Martin, 2004, 2007). We do not directly address the origin of these mysterious boutons, but along with earlier studies, our work suggests that local inputs could be a large contributor of inputs to L1 (Brown and Hestrin, 2009; Manns et al., 2004; Narayanan et al., 2015; Peng et al., 2021; Sakmann, 2017; Wozny and Williams, 2011). 
An additional feature of cortical organization is a substantial recurrent local excitatory and inhibitory connectivity (see Sachdev et al., 2012; Sherman and Usrey, 2021 for reviews). Our work suggests that local recurrent inputs are important in L1 as well. Neurons with dendrites in L1 -- i.e. L2/3 and L5 pyramidal neurons or L2/3 VIP / SST neurons -- and neurons with axons in L1 can contribute local recurrent excitation and inhibition in this layer (Larkum et al., 2018; Wozny and Williams, 2011). L1 neurons are inhibitory and could via inhibitory interactions in L1, disinhibit pyramidal neurons (Cohen-Kashi Malina et al., 2021). Our work shows that input to L1 from local L5 pyramidal neurons has specific functional consequences, it targets L1 interneurons but not the dendrites of L5 pyramidal neurons in L1. L5 pyramidal neurons do not seem to generate local recurrent excitation to other L5 pyramidal neurons via their inputs to L1. Taken together our work suggests that circuit mechanisms exist for driving local recurrent excitation and inhibition through synapses in L1 (Abs et al., 2018; Sakmann, 2017; Schuman et al., 2021; Wozny and Williams, 2011).

Long-range input to L1 is from L2/3 and L5 and cell-type specific. Our work shows that L2/3 and L5 cortical pyramidal cells are the principal cortico-cortical connection neurons that target L1. While neurons in contralateral S1, M1 and perirhinal cortices, and ipsilateral S2, M1, M2, perirhinal and visual cortices provide long-range input to L1, the bulk of the long-range input to L1 is from S2, M1, M2, visual cortices and thalamus (Figure 8C). Our work also shows that $10 \%$ to $20 \%$ of the PT and IT neurons in S2 and M1 and contralateral S1 project to L1 of S1 and that Vip and Sst neurons primarily in L2/3 target L1. While L6 cortico-cortical neurons may provide some intracortical feedback, their input to L1 is negligible. In contrast to the results with fb applied on the cortical surface, when tracer was injected into S1 cortex, an additional class of cortico-cortical projection neurons in L6 was evident, data which relate to previous studies on hierarchical models with retrograde tracers (Markov et al., 2014; Vezoli et al., 2021).

Few neurons that are presynaptic to L5 pyramidal neurons project to L1. Previous work has shown that motor cortices, POm thalamus, and perirhinal cortex project to L1 and have substantial input to L5 (Aronoff et al., 2010; Avermann et al., 2012; van der Bourg et al., 2017; Chen et al., 2013, 2015, 2016; Doron et al., 2020; Kinnischtzke et al., 2014, 2016; Yamashita et al., 2018; Zagha et al., 2013). Our work with rabies virus shows that input from higher order somatosensory cortex, S2, to L5 PT and IT neurons constitutes at more than $40 \%$ of the total long-range input to these neurons. Additionally, the rabies approach shows that some L6a and L6b neurons in S2 and M1 are presynaptic to the IT neurons. Nevertheless, even for S2, only a fraction of neurons that were presynaptic to PT and IT neurons also projected to L1. Note 
that Tlx3 and Sim1 neurons are only subclasses of all L5 neurons, consequently these connectivity numbers are likely to be underestimates.

\section{Methodological considerations}

Application of fast blue. An important potential caveat of retrograde tracing with fb is non-specific labelling of neurons under the application site: Dendrites of pyramidal neurons could potentially have uptake of fb, and/or fb could spread into L2/3 or L4. To mitigate against this, we 1) counted the labeled neurons at the application site and $>140$ microns from the application site and saw a similar pattern of local label. The proportion of local fb labeled neurons was substantially larger than the long-range input; 2) we injected fb into cortex and found a different laminar pattern of both local and long-range label; 3) we discarded brains with fb labeled neurons in VPm, which would have arisen from fb spread into L2/3 and L4.

Definition of cortical layers. While L1 and L6b are easily defined, the borders that separate the other layers from each other are not easily identifiable. Our approach was to define layers using 100/200-micron bins. This approach has the benefit of consistency and reproducibility, but there can be errors arising from brain shrinkage, brain size or selection of sections used for counting. To mitigate against this, we examined whether the proportion of $\mathrm{fb}$ labeled neurons in each layer changed, if we used a 30-micron strip at the center of the 100/200micron bin defining each layer. The proportion of fb labeled neurons in L4 changed with this approach, suggesting that some of the L4 label could be attributed to L2/3 or L5.

Rabies virus tracing. The incubation time we used here for rabies tracing (7 days) could have been too short to obtain expression in all long-range neurons (Kim et al., 2016). Furthermore, even though we have ruled out leakage of rabies virus and found little nonspecific expression (Zolnik et al., 2020), the rabies virus approach could be marginally nonspecific. Note that the questions we wanted to address with this approach -- whether neurons that project to L1 also project to L5 and whether neurons that are provide input to the apical dendrites of pyramidal cells could also generate input the cell bodies or basal dendrites and somas of these neurons -- are only partially addressed by our work.

\section{Implication}

The nub of the mystery concerning L1 can be reduced to two questions: 1) How is the input to this layer organized, and 2) What does the input do? Our work shows that L1 has some features that are common to neocortical organization. Most input to this layer is local, arising from excitatory and inhibitory neurons in the patch of cortex underlying L1. This implies that 
when feedforward, feedback, or both inputs drive somatic action potentials in L2/3 or L5, this activity is likely to directly generate synaptic activity in L1, thus likely to modulate the effect of a backpropagating action potentials that interact with contextual long-range input. Overall, our work suggests that local input to L1 may play a critical role in the information flowing through the underlying cortex. 


\section{References}

Abs, E., Poorthuis, R.B., Apelblat, D., Muhammad, K., Pardi, M.B., Enke, L., Kushinsky, D., Pu, D.-L., Eizinger, M.F., Conzelmann, K.-K., et al. (2018). Learning-Related Plasticity in Dendrite-Targeting Layer 1 Interneurons. Neuron 100, 684-699.e6.

Aertsen, A., Rotter, S., Voges, N., and Schu, A. (2010). Progress in Neurobiology A modeler ' s view on the spatial structure of intrinsic horizontal connectivity in the neocortex. 92, 277292.

Aronoff, R., Matyas, F., Mateo, C., Ciron, C., Schneider, B., and Petersen, C.C.H. (2010). Long-range connectivity of mouse primary somatosensory barrel cortex. Eur. J. Neurosci. 31, 2221-2233.

Aru, J., Suzuki, M., and Larkum, M.E. (2020). Cellular Mechanisms of Conscious Processing. Trends Cogn. Sci. 24, 814-825.

Avermann, M., Tomm, C., Mateo, C., Gerstner, W., and Petersen, C.C.H. (2012). Microcircuits of excitatory and inhibitory neurons in layer 2/3 of mouse barrel cortex. J. Neurophysiol. 107, 3116-3134.

Binzegger, T., Douglas, R.J., and Martin, K.A.C. (2004). A quantitative map of the circuit of cat primary visual cortex. J. Neurosci. 24, 8441-8453.

Boucsein, C., and Martin, P. (2011). Beyond the cortical column : abundance and physiology of horizontal connections imply a strong role for inputs from the surround. 5, 1-13.

Boucsein, C., Nawrot, M.P., Schnepel, P., and Aertsen, A. (2011). Beyond the cortical column: abundance and physiology of horizontal connections imply a strong role for inputs from the surround. Front. Neurosci. 5, 32.

van der Bourg, A., Yang, J.-W., Reyes-Puerta, V., Laurenczy, B., Wieckhorst, M., Stuttgen, M.C., Luhmann, H.J., and Helmchen, F. (2017). Layer-Specific Refinement of Sensory Coding in Developing Mouse Barrel Cortex. Cereb. Cortex 27, 4835-4850.

Brown, S.P., and Hestrin, S. (2009). Intracortical circuits of pyramidal neurons reflect their long-range axonal targets. Nature 457, 1133-1136.

Burkhalter, a (1989). Intrinsic connections of rat primary visual cortex: laminar organization of axonal projections. J. Comp. Neurol. 279, 171-186.

Cajal, S. (1899a). Estudios sobre la corteza cerebral humana I: corteza visual. Rev. Trim. Micrográf Madr. 4, 1-63.

Cajal, S. (1899b). Apuntes para el estudio estructural de la corteza visual del cerebro humano. Rev. Ibero-Americana Cienc. Méd. 1, 14. 
Cauller, L. (1995). Layer I of primary sensory neocortex: where top-down converges upon bottom-up. Behav. Brain Res. 71, 163-170.

Cauller, L.J., Clancy, B., and Connors, B.W. (1998). Backward cortical projections to primary somatosensory cortex in rats extend long horizontal axons in layer I. J. Comp. Neurol. 390, 297-310.

Chen, J.L., Carta, S., Soldado-Magraner, J., Schneider, B.L., and Helmchen, F. (2013). Behaviour-dependent recruitment of long-range projection neurons in somatosensory cortex. Nature 499, 336-340.

Chen, J.L., Margolis, D.J., Stankov, A., Sumanovski, L.T., Schneider, B.L., and Helmchen, F. (2015). Pathway-specific reorganization of projection neurons in somatosensory cortex during learning. Nat. Neurosci. 18, 1101-1108.

Chen, J.L., Voigt, F.F., Javadzadeh, M., Krueppel, R., and Helmchen, F. (2016). Long-range population dynamics of anatomically defined neocortical networks. Elife 5.

Choi, J., and Callaway, E.M. (2011). Monosynaptic inputs to ErbB4-expressing inhibitory neurons in mouse primary somatosensory cortex. J. Comp. Neurol. 519, 3402-3414.

Clancy, B., and Cauller, L.J. (1999). Widespread projections from subgriseal neurons (layer VII) to layer I in adult rat cortex. J. Comp. Neurol. 407, 275-286.

Cohen-Kashi Malina, K., Tsivourakis, E., Kushinsky, D., Apelblat, D., Shtiglitz, S., Zohar, E., Sokoletsky, M., Tasaka, G.-I., Mizrahi, A., Lampl, I., et al. (2021). NDNF interneurons in layer 1 gain-modulate whole cortical columns according to an animal's behavioral state. Neuron.

Cruikshank, S.J., Ahmed, O.J., Stevens, T.R., Patrick, S.L., Gonzalez, A.N., Elmaleh, M., and Connors, B.W. (2012). Thalamic control of layer 1 circuits in prefrontal cortex. J. Neurosci. 32, 17813-17823.

DeFelipe, J. (1997). Types of neurons, synaptic connections and chemical characteristics of cells immunoreactive for calbindin-D28K, parvalbumin and calretinin in the neocortex. J. Chem. Neuroanat. 14, 1-19.

DeFelipe, J., and Fariñas, I. (1992). The pyramidal neuron of the cerebral cortex: morphological and chemical characteristics of the synaptic inputs. Prog. Neurobiol. 39, 563607.

DeFelipe, J., Jones, EG, Ramon y Cajal, SY (1988). Cajal on the Cerebral Cortex: An Annotated Translation of the Complete Writings (History of Neuroscience, Band 1). Oxford University Press 1988.

Doron, G., Shin, J.N., Takahashi, N., Drüke, M., Bocklisch, C., Skenderi, S., de Mont, L., Toumazou, M., Ledderose, J., Brecht, M., et al. (2020). Perirhinal input to neocortical layer 1 
controls learning. Science 370.

Douglas, R.J., and Martin, K.A.C. (2004). Neuronal circuits of the neocortex. Annu. Rev. Neurosci. 27, 419-451.

Douglas, R.J., and Martin, K.A.C. (2007). Perspective Mapping the Matrix: The Ways of Neocortex. 226-238.

Feldmeyer, D., Lübke, J., and Sakmann, B. (2006). Efficacy and connectivity of intracolumnar pairs of layer 2/3 pyramidal cells in the barrel cortex of juvenile rats. J. Physiol. 575, 583-602. Felleman, D.J., and Van Essen, D.C. (1991). Distributed hierarchical processing in the primate cerebral cortex. Cereb. Cortex 1, 1-47.

Gabbott, P.L., and Somogyi, P. (1986). Quantitative distribution of GABA-immunoreactive neurons in the visual cortex (area 17) of the cat. Exp. Brain Res. 61, 323-331.

Gămănuţ, R., Kennedy, H., Toroczkai, Z., Ercsey-Ravasz, M., Van Essen, D.C., Knoblauch, K., and Burkhalter, A. (2018). The Mouse Cortical Connectome, Characterized by an UltraDense Cortical Graph, Maintains Specificity by Distinct Connectivity Profiles. Neuron 97, 698-715.e10.

Gerfen, C.R., Paletzki, R., and Heintz, N. (2013). GENSAT BAC cre-recombinase driver lines to study the functional organization of cerebral cortical and basal ganglia circuits. Neuron 80 , 1368-1383.

Gong, S., Zheng, C., Doughty, M.L., Losos, K., Didkovsky, N., Schambra, U.B., Nowak, N.J., Joyner, A., Leblanc, G., Hatten, M.E., et al. (2003). A gene expression atlas of the central nervous system based on bacterial artificial chromosomes. Nature 425, 917-925.

Heuer, H., Christ, S., Friedrichsen, S., Brauer, D., Winckler, M., Bauer, K., and Raivich, G. (2003). Connective tissue growth factor: a novel marker of layer VII neurons in the rat cerebral cortex. Neuroscience 119, 43-52.

Hoerder-Suabedissen, A., Hayashi, S., Upton, L., Nolan, Z., Casas-Torremocha, D., Grant, E., Viswanathan, S., Kanold, P.O., Clasca, F., Kim, Y., et al. (2018). Subset of Cortical Layer 6b Neurons Selectively Innervates Higher Order Thalamic Nuclei in Mice. Cereb. Cortex 28, 1882-1897.

Hubel, D.H. (1982). Exploration of the primary visual cortex, 1955-78. Nature 299, 515-524. Ibrahim, L.A., Schuman, B., Bandler, R., Rudy, B., and Fishell, G. (2020). Mining the jewels of the cortex’s crowning mystery. Curr. Opin. Neurobiol. 63, 154-161.

Ito, M., Kato, M., and Kawabata, M. (1998). Premature bifurcation of the apical dendritic trunk of vibrissa-responding pyramidal neurones of X-irradiated rat neocortex. J. Physiol. 512 ( Pt 2), 543-553. 
Karimi, A., Odenthal, J., Drawitsch, F., Boergens, K.M., and Helmstaedter, M. (2020). Celltype specific innervation of cortical pyramidal cells at their apical dendrites. Elife 9.

Keizer, K., Kuypers, H.G., Huisman, A.M., and Dann, O. (1983). Diamidino yellow dihydrochloride (DY . 2HCl); a new fluorescent retrograde neuronal tracer, which migrates only very slowly out of the cell. Exp. Brain Res. 51, 179-191.

Kim, E.J., Juavinett, A.L., Kyubwa, E.M., Jacobs, M.W., and Callaway, E.M. (2015). Three Types of Cortical Layer 5 Neurons That Differ in Brain-wide Connectivity and Function. Neuron 88, 1253-1267.

Kim, E.J., Jacobs, M.W., Ito-Cole, T., and Callaway, E.M. (2016). Improved Monosynaptic Neural Circuit Tracing Using Engineered Rabies Virus Glycoproteins. Cell Rep. 15, 692-699. Kinnischtzke, A.K., Simons, D.J., and Fanselow, E.E. (2014). Motor cortex broadly engages excitatory and inhibitory neurons in somatosensory barrel cortex. Cereb. Cortex 24, 22372248.

Kinnischtzke, A.K., Fanselow, E.E., and Simons, D.J. (2016). Target-specific M1 inputs to infragranular S1 pyramidal neurons. J. Neurophysiol. 116, 1261-1274.

Kuypers, H.G., Bentivoglio, M., Catsman-Berrevoets, C.E., and Bharos, A.T. (1980). Double retrograde neuronal labeling through divergent axon collaterals, using two fluorescent tracers with the same excitation wavelength which label different features of the cell. Exp. Brain Res. 40, 383-392.

Larkum, M. (2013). A cellular mechanism for cortical associations: An organizing principle for the cerebral cortex. Trends Neurosci. 36, 141-151.

Larkum, M.E., Petro, L.S., Sachdev, R.N.S., and Muckli, L. (2018). A Perspective on Cortical Layering and Layer-Spanning Neuronal Elements. Front. Neuroanat. 12, 56.

Lee, S., Hjerling-Leffler, J., Zagha, E., Fishell, G., and Rudy, B. (2010). The largest group of superficial neocortical GABAergic interneurons expresses ionotropic serotonin receptors. J. Neurosci. 30, 16796-16808.

Luo, L., Callaway, E.M., and Svoboda, K. (2008). Genetic Dissection of Neural Circuits. Neuron 57, 634-660.

Manns, I.D., Sakmann, B., and Brecht, M. (2004). Sub- and suprathreshold receptive field properties of pyramidal neurones in layers $5 \mathrm{~A}$ and $5 \mathrm{~B}$ of rat somatosensory barrel cortex. $\mathrm{J}$. Physiol. 556, 601-622.

Marín-Padilla, M. (1998). Cajal-Retzius cells and the development of the neocortex. Trends Neurosci. 21, 64-71.

Markov, N.T., Vezoli, J., Chameau, P., Falchier, A., Quilodran, R., Huissoud, C., Lamy, C., 
Misery, P., Giroud, P., Ullman, S., et al. (2014). Anatomy of hierarchy: Feedforward and feedback pathways in macaque visual cortex. J. Comp. Neurol. 522, 225-259.

Markwardt, M.L., Kremers, G.-J., Kraft, C.A., Ray, K., Cranfill, P.J.C., Wilson, K.A., Day, R.N., Wachter, R.M., Davidson, M.W., and Rizzo, M.A. (2011). An improved cerulean fluorescent protein with enhanced brightness and reduced reversible photoswitching. PLoS One 6, e17896.

Mason, A., and Larkman, A. (1990). Correlations between morphology and electrophysiology of pyramidal neurons in slices of rat visual cortex. II. Electrophysiology. J. Neurosci. 10, 1415-1428.

Masurkar, A. V, Srinivas, K. V, Brann, D.H., Warren, R., Lowes, D.C., and Siegelbaum, S.A. (2017). Medial and Lateral Entorhinal Cortex Differentially Excite Deep versus Superficial CA1 Pyramidal Neurons. Cell Rep. 18, 148-160.

Mease, R.A., Sumser, A., Sakmann, B., and Groh, A. (2016). Corticothalamic Spike Transfer via the L5B-POm Pathway in vivo. Cereb. Cortex 26, 3461-3475.

Narayanan, R.T., Egger, R., Johnson, A.S., Mansvelder, H.D., Sakmann, B., de Kock, C.P.J., and Oberlaender, M. (2015). Beyond Columnar Organization: Cell Type- and Target LayerSpecific Principles of Horizontal Axon Projection Patterns in Rat Vibrissal Cortex. Cereb. Cortex 25, 4450-4468.

Narayanan, R.T., Udvary, D., and Oberlaender, M. (2017). Cell Type-Specific Structural Organization of the Six Layers in Rat Barrel Cortex. Front. Neuroanat. 11, 91.

Oberlaender, M., Boudewijns, Z.S.R.M., Kleele, T., Mansvelder, H.D., Sakmann, B., and de Oberlaender, M., De Kock, C.P.J., Bruno, R.M., Ramirez, A., Meyer, H.S., Dercksen, V.J., Helmstaedter, M., and Sakmann, B. (2012). Cell type-specific three-dimensional structure of thalamocortical circuits in a column of rat vibrissal cortex. Cereb. Cortex 22, 2375-2391.

Oda, S., Kishi, K., Yang, J., Chen, S., Yokofujita, J., Igarashi, H., Tanihata, S., and Kuroda, M. (2004). Thalamocortical projection from the ventral posteromedial nucleus sends its collaterals to layer I of the primary somatosensory cortex in rat. Neurosci. Lett. 367, 394-398. Ohno, S., Kuramoto, E., Furuta, T., Hioki, H., Tanaka, Y.R., Fujiyama, F., Sonomura, T., Uemura, M., Sugiyama, K., and Kaneko, T. (2012). A morphological analysis of thalamocortical axon fibers of rat posterior thalamic nuclei: a single neuron tracing study with viral vectors. Cereb. Cortex 22, 2840-2857.

Osakada, F., and Callaway, E.M. (2013). Design and generation of recombinant rabies virus vectors. Nat. Protoc. 8, 1583-1601.

Peng, H., Xie, P., Liu, L., Kuang, X., Wang, Y., Qu, L., Gong, H., Jiang, S., Li, A., Ruan, Z., 
et al. (2021). Morphological diversity of single neurons in molecularly defined cell types. Nature 598, 174-181.

Petreanu, L., Huber, D., Sobczyk, A., and Svoboda, K. (2007). Channelrhodopsin-2-assisted circuit mapping of long-range callosal projections. Nat. Neurosci. 10, 663-668.

Ramaswamy, S., Hill, S.L., King, J.G., Schürmann, F., Wang, Y., and Markram, H. (2012). Intrinsic morphological diversity of thick-tufted layer 5 pyramidal neurons ensures robust and invariant properties of in silico synaptic connections. J. Physiol. 590, 737-752.

Rubio-Garrido, P., Pérez-De-Manzo, F., Porrero, C., Galazo, M.J., and Clascá, F. (2009). Thalamic input to distal apical dendrites in neocortical layer 1 is massive and highly convergent. Cereb. Cortex 19, 2380-2395.

Rudy, B., Fishell, G., Lee, S., and Hjerling-Leffler, J. (2011). Three groups of interneurons account for nearly $100 \%$ of neocortical GABAergic neurons. Dev. Neurobiol. 71, 45-61.

Sachdev, R.N.S., Krause, M.R., and Mazer, J. a. (2012). Surround suppression and sparse coding in visual and barrel cortices. Front. Neural Circuits 6, 1-14.

Sakmann, B. (2017). From single cells and single columns to cortical networks: dendritic excitability, coincidence detection and synaptic transmission in brain slices and brains. Exp. Physiol. 102, 489-521.

Schuman, B., Machold, R.P., Hashikawa, Y., Fuzik, J., Fishell, G.J., and Rudy, B. (2019). Four Unique Interneuron Populations Reside in Neocortical Layer 1. J. Neurosci. 39, 125-139.

Schuman, B., Dellal, S., Prönneke, A., Machold, R., and Rudy, B. (2021). Neocortical Layer 1: An Elegant Solution to Top-Down and Bottom-Up Integration. Annu. Rev. Neurosci.

Shephert, G.M. (2004). Synaptic Organization of the brain. Oxford University Press 2004.

Shepherd, G.M. (2011). The microcircuit concept applied to cortical evolution: from threelayer to six-layer cortex. Front. Neuroanat. 5, 30.

Sherman, S.M., and Usrey, W.M. (2021). Cortical control of behavior and attention from an evolutionary perspective. Neuron.

Sun, Y., Nguyen, A.Q., Nguyen, J.P., Le, L., Saur, D., Choi, J., Callaway, E.M., and Xu, X. (2014). Cell-type-specific circuit connectivity of hippocampal CA1 revealed through Credependent rabies tracing. Cell Rep. 7, 269-280.

Suzuki, M., and Larkum, M.E. (2020). General Anesthesia Decouples Cortical Pyramidal Neurons. Cell 180, 666-676.e13.

Takahashi, N., Oertner, T.G., Hegemann, P., and Larkum, M.E. (2016). Active cortical dendrites modulate perception. Science 354, 1587-1590.

Takahashi, N., Ebner, C., Sigl-Glöckner, J., Moberg, S., Nierwetberg, S., and Larkum, M.E. 
(2020). Active dendritic currents gate descending cortical outputs in perception. Nat. Neurosci. 23, 1277-1285.

Taniguchi, H., He, M., Wu, P., Kim, S., Paik, R., Sugino, K., Kvitsiani, D., Fu, Y., Lu, J., Lin, Y., et al. (2011). A resource of Cre driver lines for genetic targeting of GABAergic neurons in cerebral cortex. Neuron 71, 995-1013.

Veinante, P., and Deschênes, M. (2003). Single-cell study of motor cortex projections to the barrel field in rats. J. Comp. Neurol. 464, 98-103.

Vezoli, J., Magrou, L., Goebel, R., Wang, X.-J., Knoblauch, K., Vinck, M., and Kennedy, H. (2021). Cortical hierarchy, dual counterstream architecture and the importance of top-down generative networks. Neuroimage 225, 117479.

White EL (1989). Cortical Circuits. Synaptic Organization of the Cerebral Cortex. Birkhäuser Boston Basel Berlin 1989.

Wickersham, I.R., Lyon, D.C., Barnard, R.J.O., Mori, T., Finke, S., Conzelmann, K.K., Young, J. a T., and Callaway, E.M. (2007). Monosynaptic Restriction of Transsynaptic Tracing from Single, Genetically Targeted Neurons. Neuron 53, 639-647.

Wickersham, I.R., Sullivan, H.A., and Seung, H.S. (2013). Axonal and subcellular labelling using modified rabies viral vectors. Nat. Commun. 4, 2332.

Wozny, C., and Williams, S.R. (2011). Specificity of synaptic connectivity between layer 1 inhibitory interneurons and layer $2 / 3$ pyramidal neurons in the rat neocortex. Cereb. Cortex 21, 1818-1826.

Yamashita, T., Vavladeli, A., Pala, A., Galan, K., Crochet, S., Petersen, S.S.A., and Petersen, C.C.H. (2018). Diverse Long-Range Axonal Projections of Excitatory Layer 2/3 Neurons in Mouse Barrel Cortex. Front. Neuroanat. 12, 33.

Yamawaki, N., Raineri Tapies, M.G., Stults, A., Smith, G.A., and Shepherd, G.M. (2021). Circuit organization of the excitatory sensorimotor loop through hand/forelimb S1 and M1. Elife 10.

Zagha, E., Casale, A.E., Sachdev, R.N.S., McGinley, M.J., and McCormick, D. a. (2013). Motor cortex feedback influences sensory processing by modulating network state. Neuron 79, 567-578.

Zolnik, T.A., Ledderose, J., Toumazou, M., Trimbuch, T., Oram, T., Rosenmund, C., Eickholt, B.J., Sachdev, R.N.S., and Larkum, M.E. (2020). Layer 6b Is Driven by Intracortical LongRange Projection Neurons. Cell Rep. 30, 3492-3505.e5. 


\section{Figures}
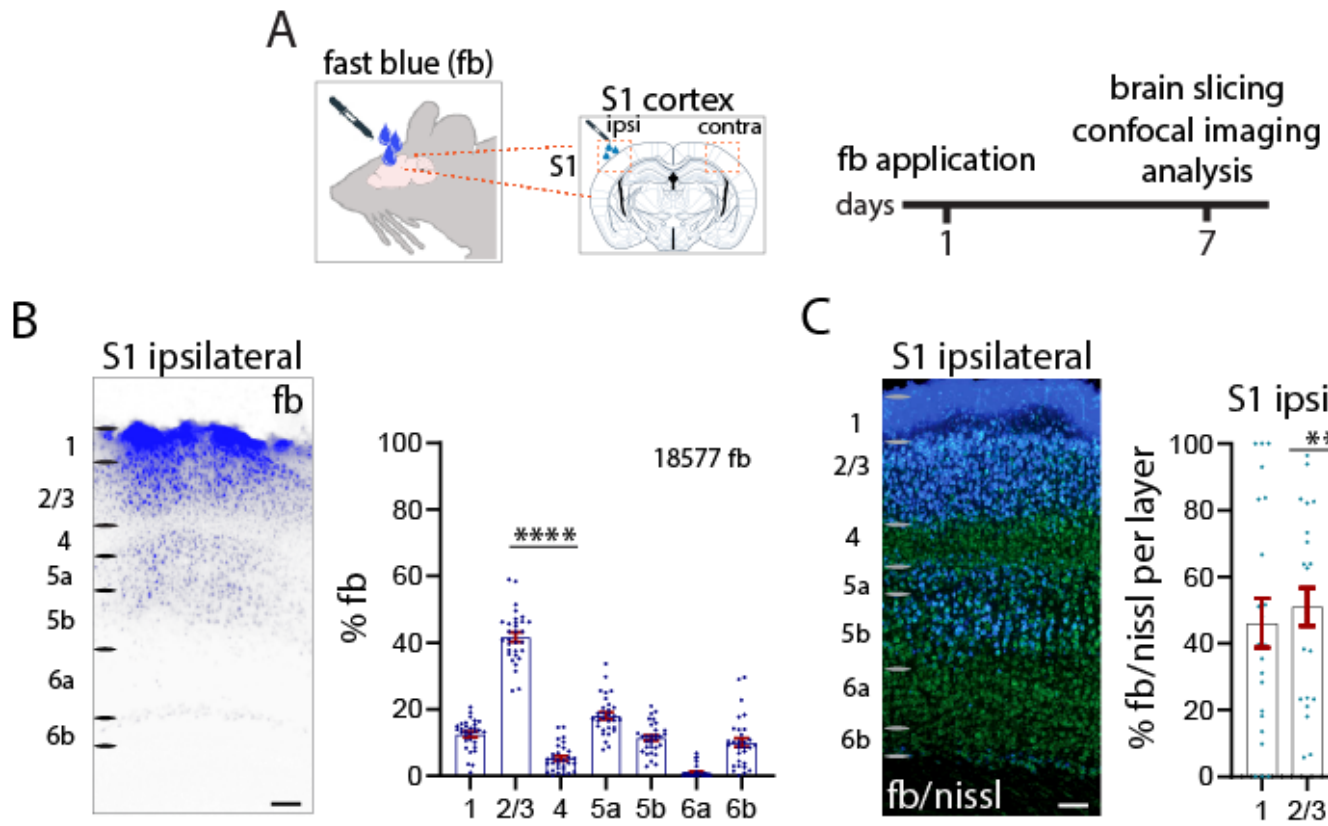

C
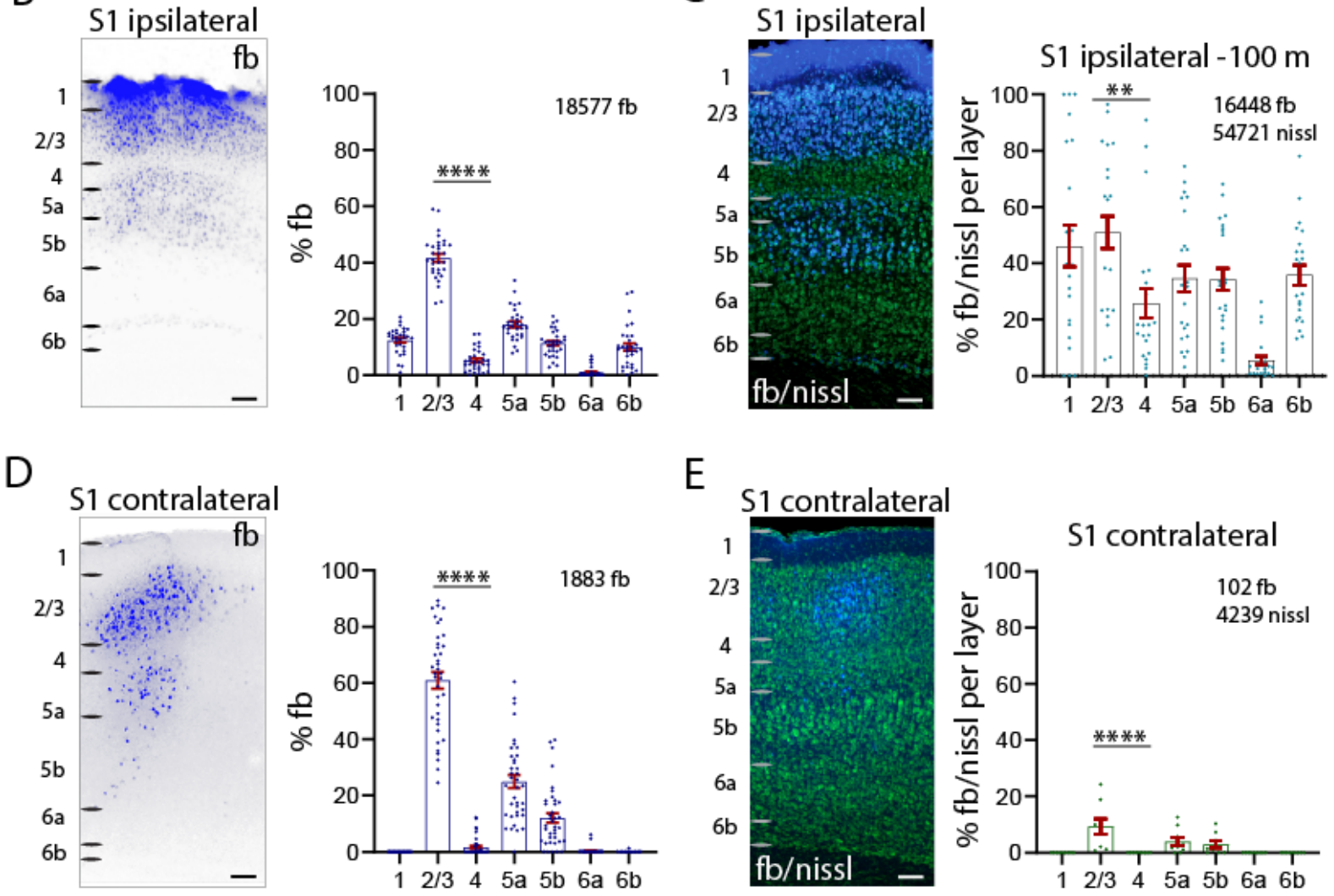

E

F
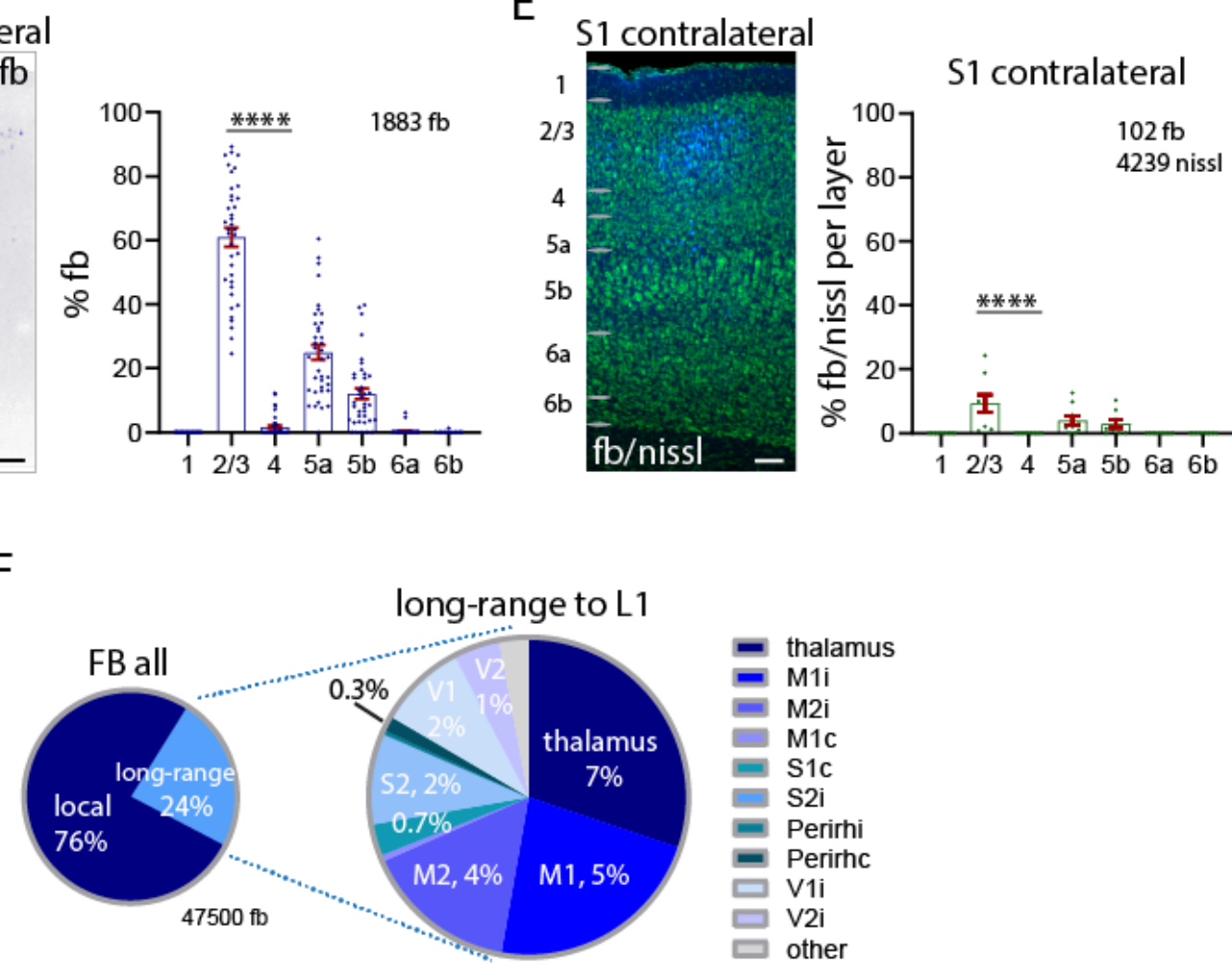

Figure 1. Input to S1 L1. (A) Schematic of experiment. Retrograde tracer fb was applied on L1 of S1 cortex and fb expressing cells were counted seven days after incubation. (B) Fast blue labeled neurons at application site. Uptake was evident across all cortical layers, with prominent uptake in L2/3 and L5. The tabulation of fb labeled neurons in different lamina under the application site is shown in the bar graph. (C) Percentage of L1 projecting fb labeled neurons in each layer. Nissl stained and double labeled neurons were counted to obtain a 
percentage of labeled neurons in each lamina. L2/3 and L5 had the highest percentage of double labeled neurons. (D) Laminar pattern of contralateral S1 projections to L1. The contralateral projection to L1 arises almost exclusively from neurons in L2/3 and L5. (E) Percentage of fb labeled neurons in each layer of contralateral S1. Nine percent of L2/3 neurons in contralateral S1 project to L1. (F) Proportion of local and long-range input and classification of long-range input to L1. The bulk of input to L1 was from local neurons (76\%), with 24\% of the input arising from long-range sources. Thalamus (including POm, zona incertai), ipsilateral and contralateral motor cortices, contralateral S1, ipsilateral S2, bilateral perirhinal (perirh), and ipsilateral visual cortices all provide input to L1 Abbreviations in F: i, ipsi; c, contralateral. Each dot in the graphs represents one brain section. Total numbers of neurons counts are shown in each panel. Statistical analysis with one-way ANOVA, Bonferroni post-hoc test, $* * * * \mathrm{p}<0.0001$. Analysis details in Tables 1-1, 1-2. Scale bars $100 \mu \mathrm{m}$. 

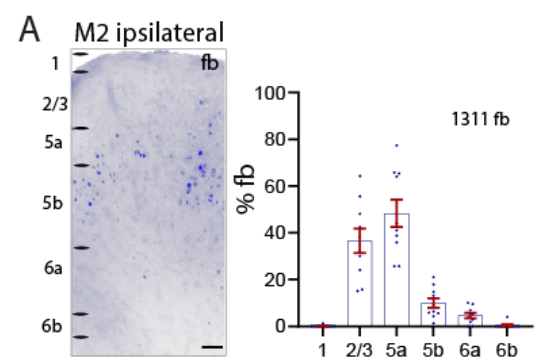

M2 ipsilateral

B $\quad$ M1 ipsilateral
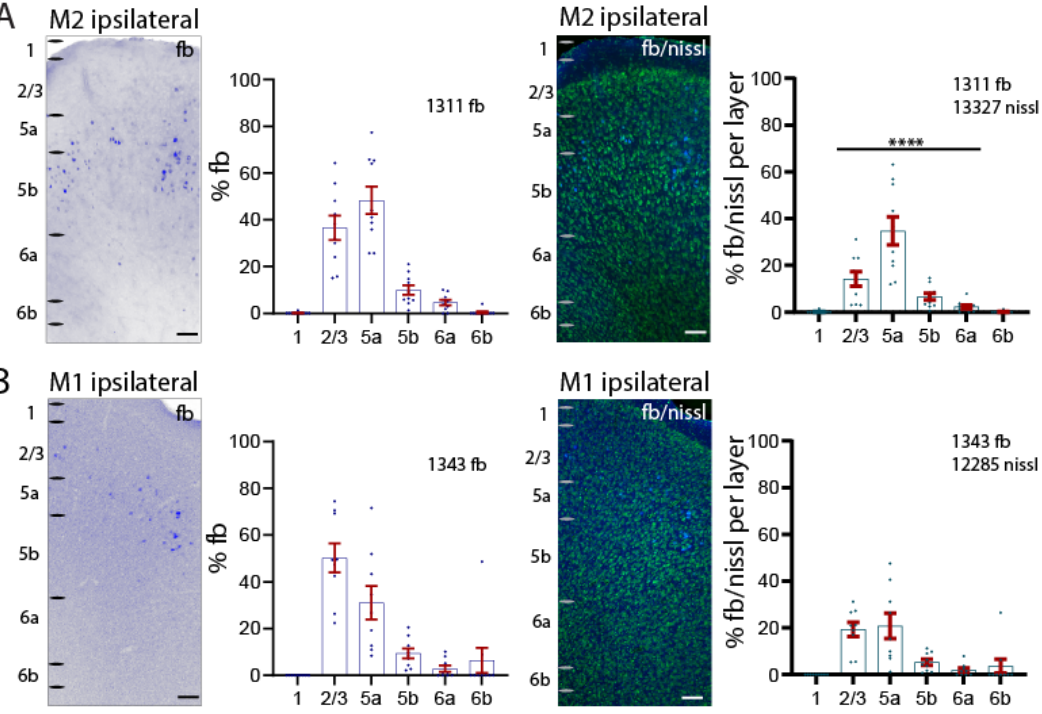

M1 ipsilateral
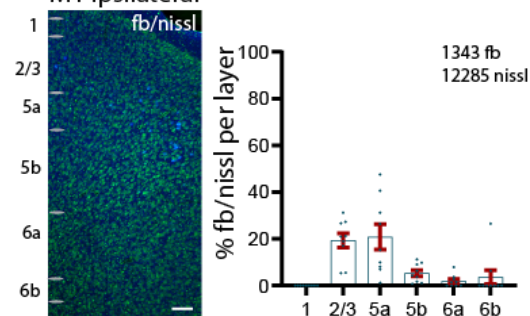

C S2 ipsilateral
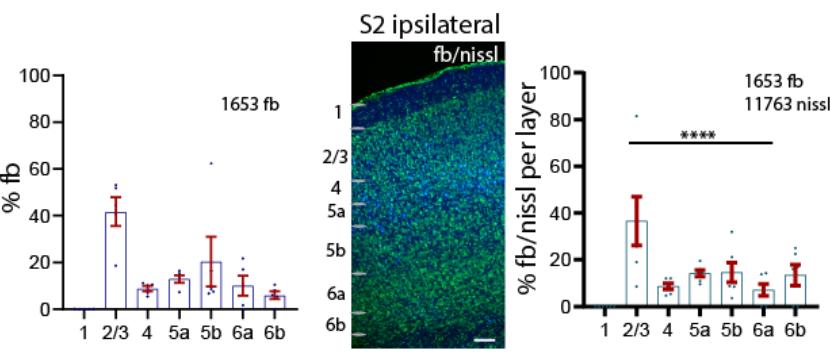

D V1/N2 ipsilateral

V1/V2 ipsilateral
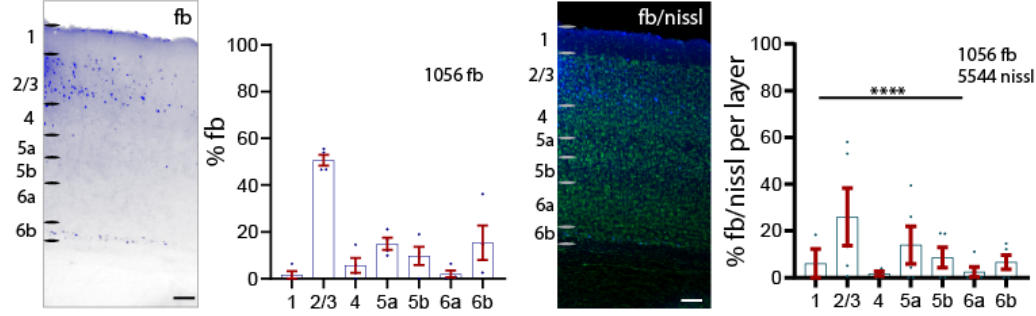

E perihinalipsilateral
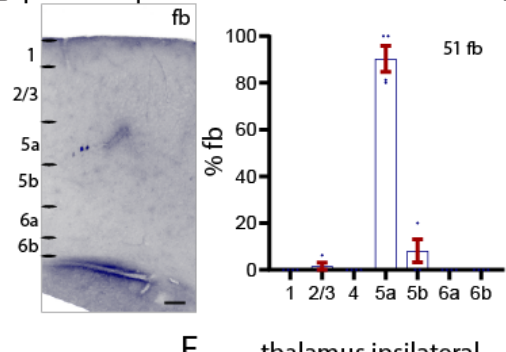

perirhinal contralateral
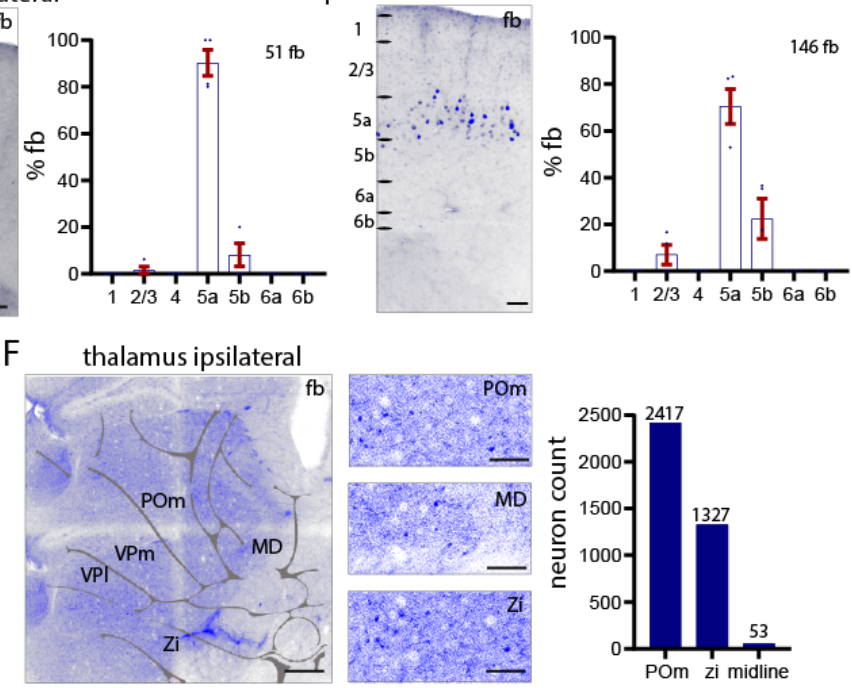

Figure 2. Long-range input to S1 L1 from other cortical areas. (A-D) Percentage of total $\mathrm{fb}$ labeled neurons (left), and percentage of labeled neurons in relation to nissl in each layer (right). (A) M2 cortex, (B) M1 cortex, (C) S2 cortex, (D) visual cortices, (E) perirhinal cortices ipsilateral and contralateral. Counts of fb labeled neurons ipsilateral to application site (left) 
and contralateral (right). Quantification of the laminar pattern of long-range input to L1 shows the highest percentage of fb labeled neurons in cortical L2/3 and L5a. (F) Fast blue label (total numbers) in POm thalamus, zona incerta and midline thalamus shows that these three subcortical nuclei have projections to L1. Abbreviations in F: MD, midline; PoM, posteromedial complex of thalamus; VPl, ventrolateral nucleus of thalamus; VPm, ventromedial nucleus of thalamus; $\mathrm{Zi}$, zona inserta. Each dot in the graphs represents one brain section. Total numbers of neurons counted in each brain area are shown in each panel. Data from four brains. Statistical analysis with one-way ANOVA, Bonferroni post-hoc test, $* * * * \mathrm{p}<0.0001$. Analysis details in Table 2-1. Scale bars in A-E $100 \mu \mathrm{m}$, in F $500 \mu \mathrm{m}$, in zoomins $50 \mu \mathrm{m}$. 


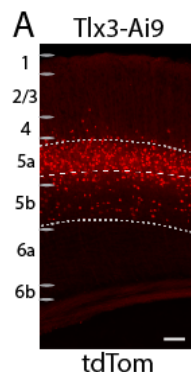

tdTom
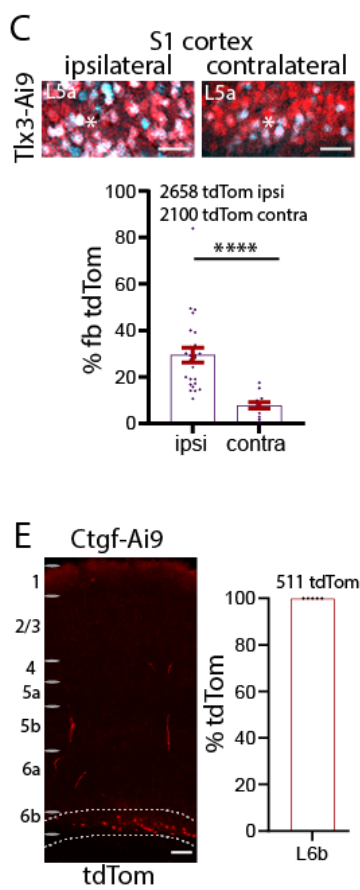

G

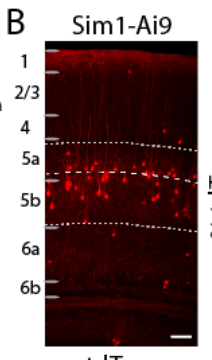

tdTom

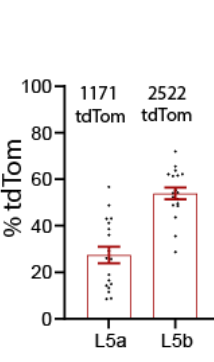

Sim1-Ai9

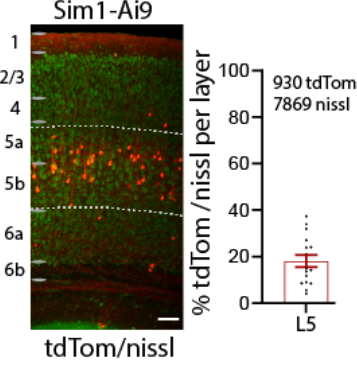

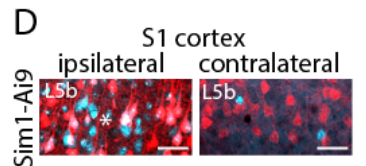

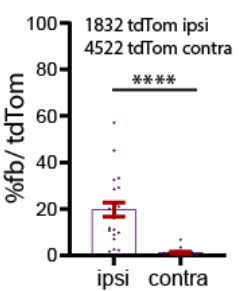

Motor cortex S2 cortex ipsilateral ipsilateral
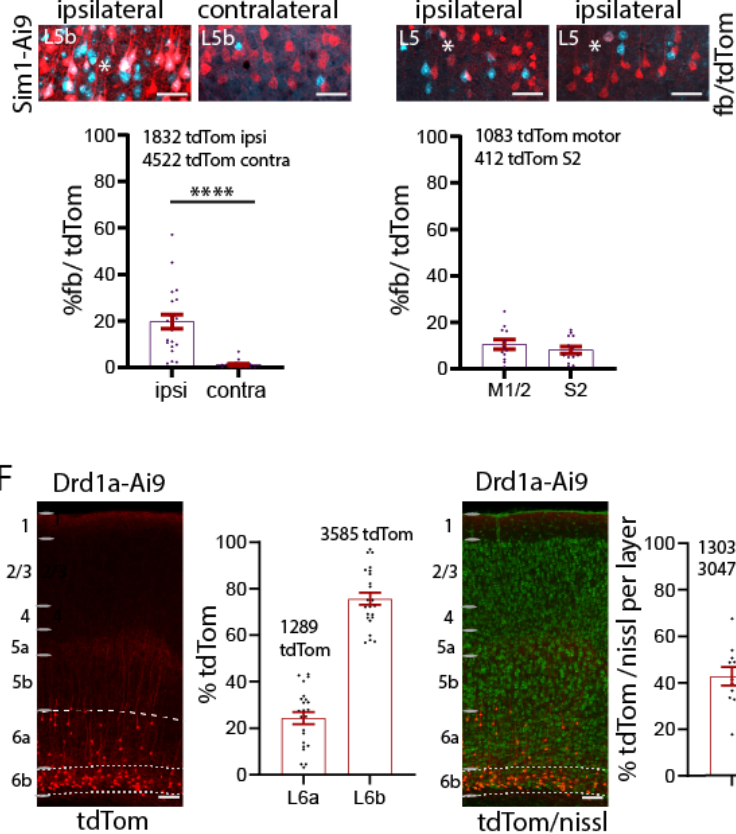
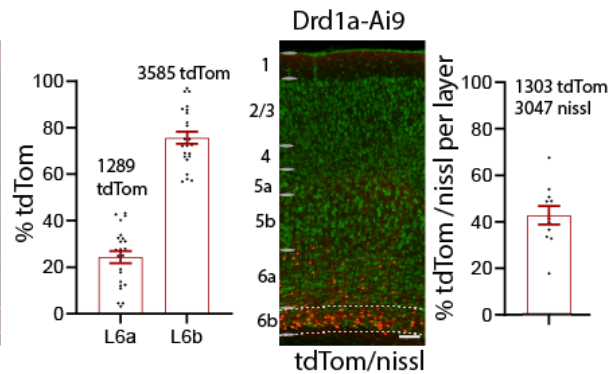

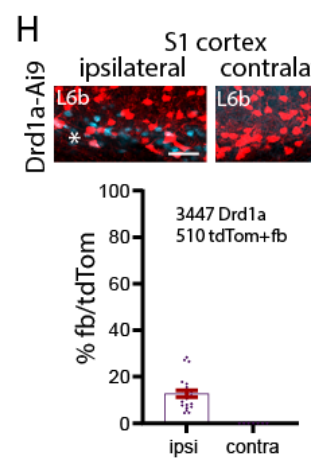

Figure 3. Input from classes of L5 and L6b cells to L1. (A) Images of sections showing intratelencephalic Tlx3-Cre/ tdTomato positive and the nissl stained neurons and quantification. Sixty-seven percent of tdTomato positive cells in this line were in L5a. Fortytwo percent of all L5 cells were tdTomato positive. (B) Sections showing pyramidal tract Sim1Cre/ tdTomato positive and nissl stained neurons and quantification. In this line, $18 \%$ of L5 neurons were tdTomato positive. (C) Example images of fb uptake in Tlx3-Cre brain. Ipsilaterally, 29\% of Tlx3 and contralaterally, 8\% of Tlx3 neurons in S1 project to L1. Tlx3 neurons in motor and S2 cortices also project to L1. Tlx3 neurons in motor cortices and S2 cortices also project to L1. (D) Example images of fb uptake in Sim1-Cre mice. Ipsilaterally, $20 \%$ of Sim1 neurons project to L1, and contralaterally, few Sim1 neurons in S1 project to L1. Sim1 neurons in motor cortices and S2 cortices also project to L1. (E) Proportion of L6b neurons labeled in Ctgf-Cre/ tdTomato positive and nissl stained neurons. All Ctgf neurons are located in L6b. Fifteen percent of tdTomato positive cells in this line were in L6b. (F) In the 
Drd1a-Cre line 76\% of tdTomato positive cells are located in L6b, the other remaining in L6a. Forty-two percent of all L6b neurons in L6b is Drd1a positive. (G) Percentages of fb expressing neurons in Ctgf L6b line. A large proportion of L6b Ctgf positive neurons project to L1. (H) Percentages of $\mathrm{fb}$ expressing neurons in L6b in the Drd1a line. Only 13\% of L6b Drd1a neurons project to L1. Each dot in the graphs represents one brain section. Total number of neurons counted are shown in each panel. Statistical analysis with one-way ANOVA ****p $<0.0001$. Analysis details in Table 3-1. Fast blue pseudo colored in cyan. Scale bars in A, B, E, F 100 $\mu \mathrm{m}$, in C, D, G, H $50 \mu \mathrm{m}$. 


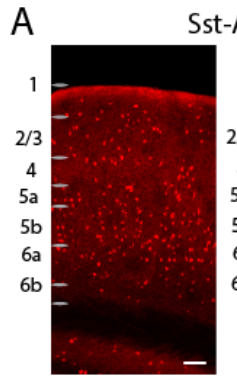

tdTom

C

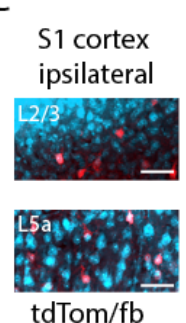

tdTom/nissl

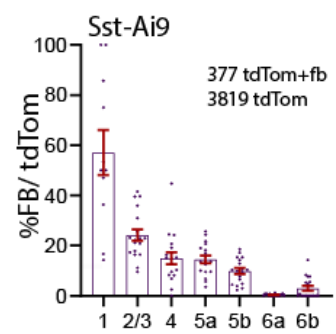

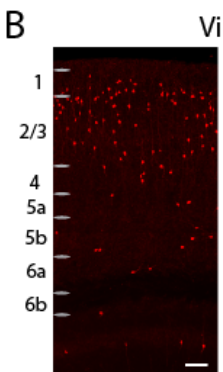

tdTom

D

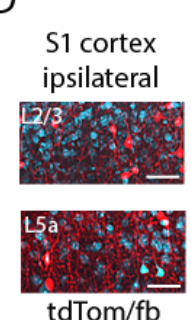

460 tdTom 9780 niss

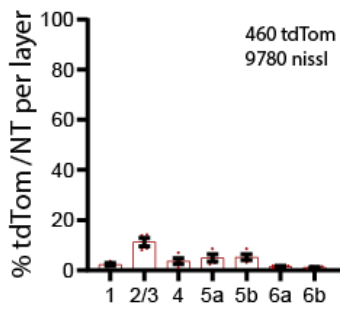

tdTom/nissl

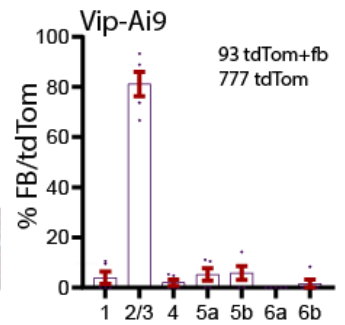

Figure 4. Inhibitory input to L1. (A) Pattern of Sst input. Quantification of laminar pattern of Sst positive neurons with nissl stain. (B) Pattern of Vip input to L1. Quantification of laminar pattern shows percentages of Vip neurons. (C) Example image and quantification of Sst-Cre/ tdTomato in S1 cortex after fb application. Neurons in all layers take up $\mathrm{fb}$ on the ipsilateral side. (D) Example image of Vip-Cre/ tdTomato in S1 cortex after fb application. More than $80 \%$ of L2/3 Vip neurons take up fb. Each dot in the graphs represents one brain section. Total number of neurons counted in each Cre line are shown in each panel. Analysis details in Table 4-1. Scale bars in A and B, $100 \mu \mathrm{m}$, in $C$ and $D, 50 \mu \mathrm{m}$. 


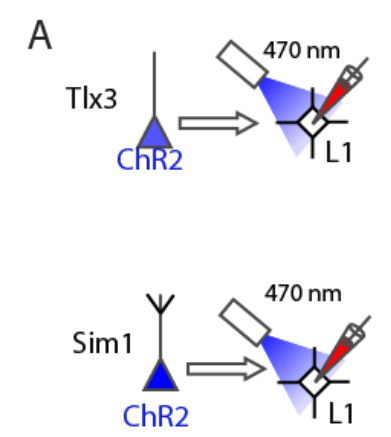

B
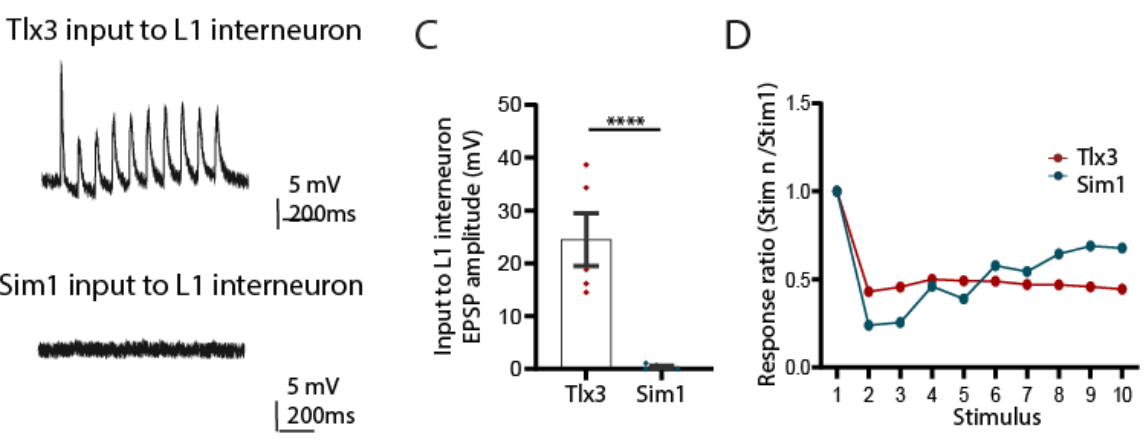

$\mathrm{E}$

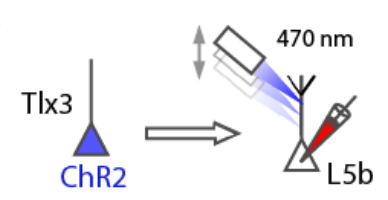

$\mathrm{F}$
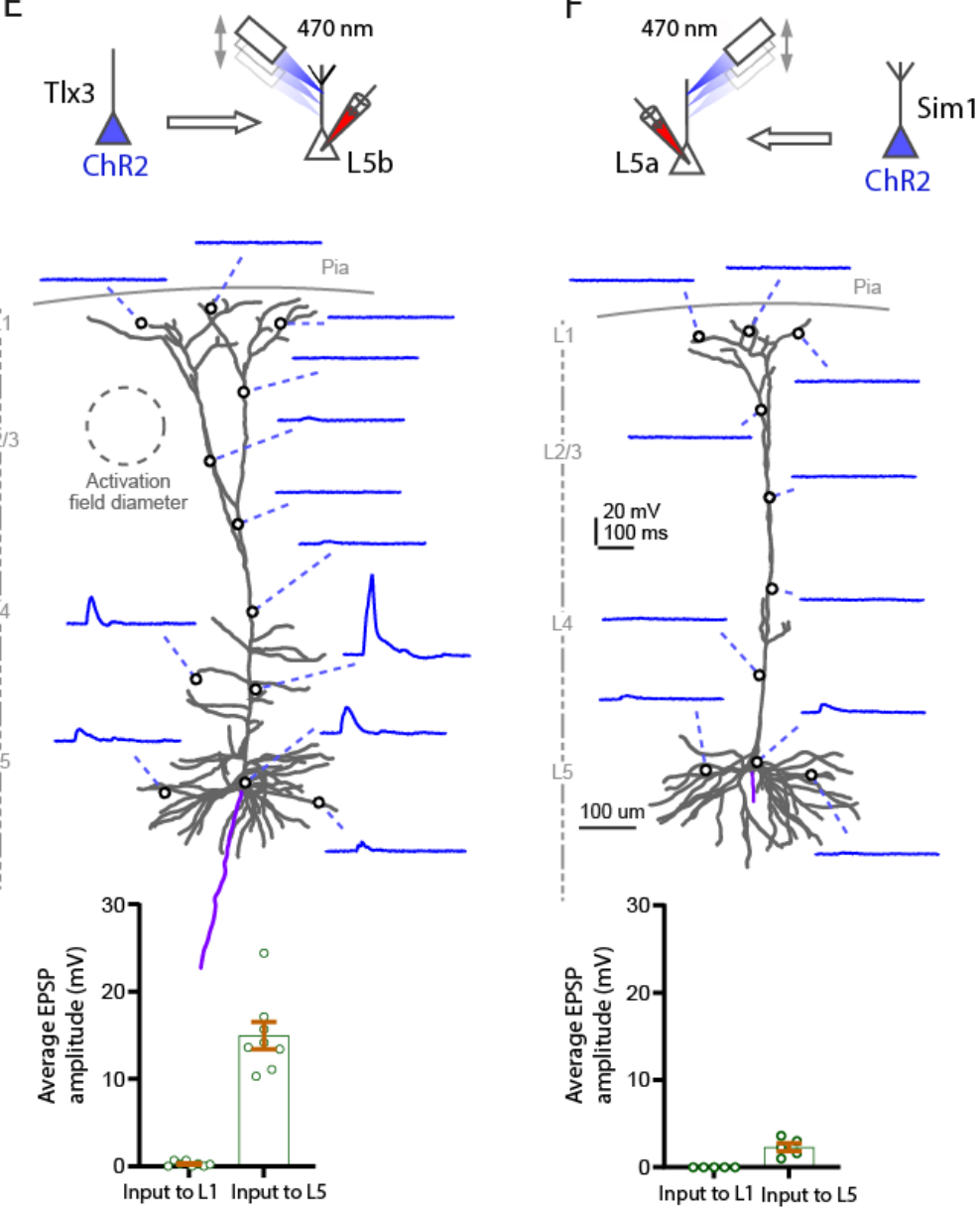

Figure 5. Functional synaptic input to L1 from L5 Tlx3 and Sim1 neurons in S1 cortex. (A) Experimental setup to test the input from L5 Tlx3-Cre and L5 Sim1-Cre neurons onto L1 interneurons. (B) Example recordings of input to L1 interneurons from Tlx3 (top) and Sim1 (bottom) neurons. (C) Group data of evoked EPSPs in L1 interneurons (Tlx3, n=5; Sim1, n=5). (D) Short-term dynamics of evoked EPSPs in L1 interneurons (10 Hz; Tlx3, n=5; Sim1, n=2). (E, F) Top, experimental setup to test the input from L5 IT (Tlx3) (E) and L5 PT (Sim1) (F) neurons onto subcellular spots along L5b PT $(n=8)$ and L5a IT $(n=5)$ neurons, respectively. Middle, example neurons and their responses to light spot illumination in the indicated locations on each neuron (E, L5b neuron, input from Tlx3; F, L5a neuron, input from Sim1). Bottom, group data comparing the input to L1 (apical tufts) and L5 (somatic region). 
A

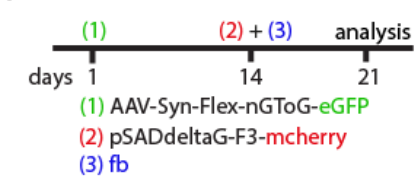

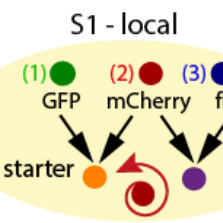

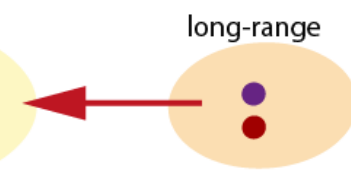

B
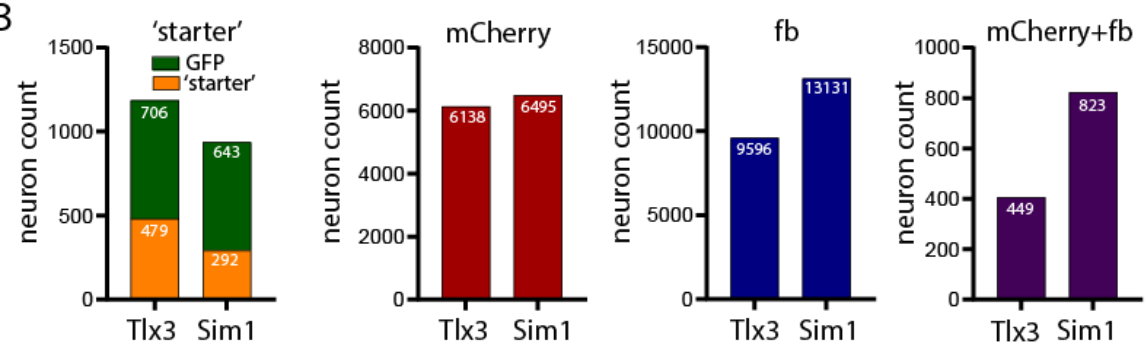

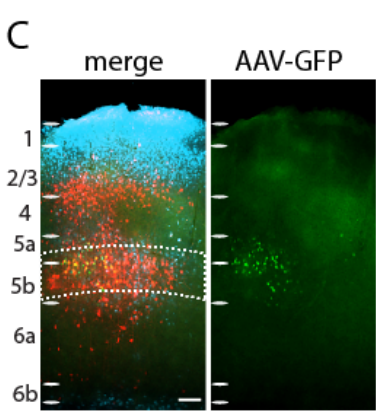

Tlx3-Cre RV-mCherry

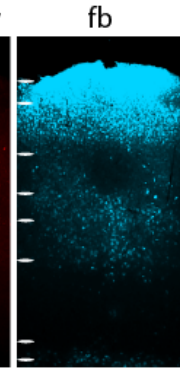
'starter'
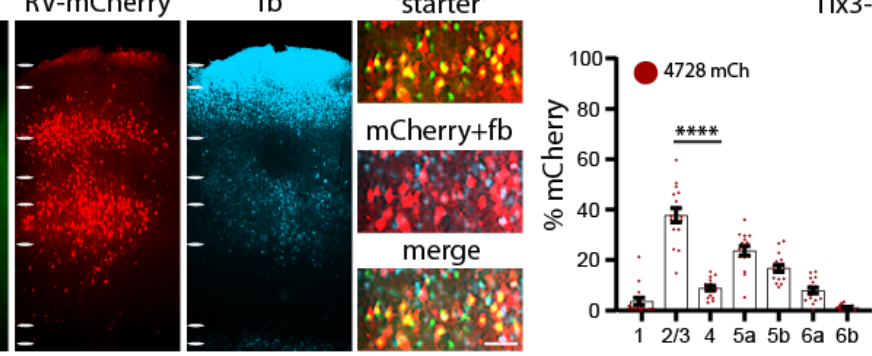

TIx3-Cre
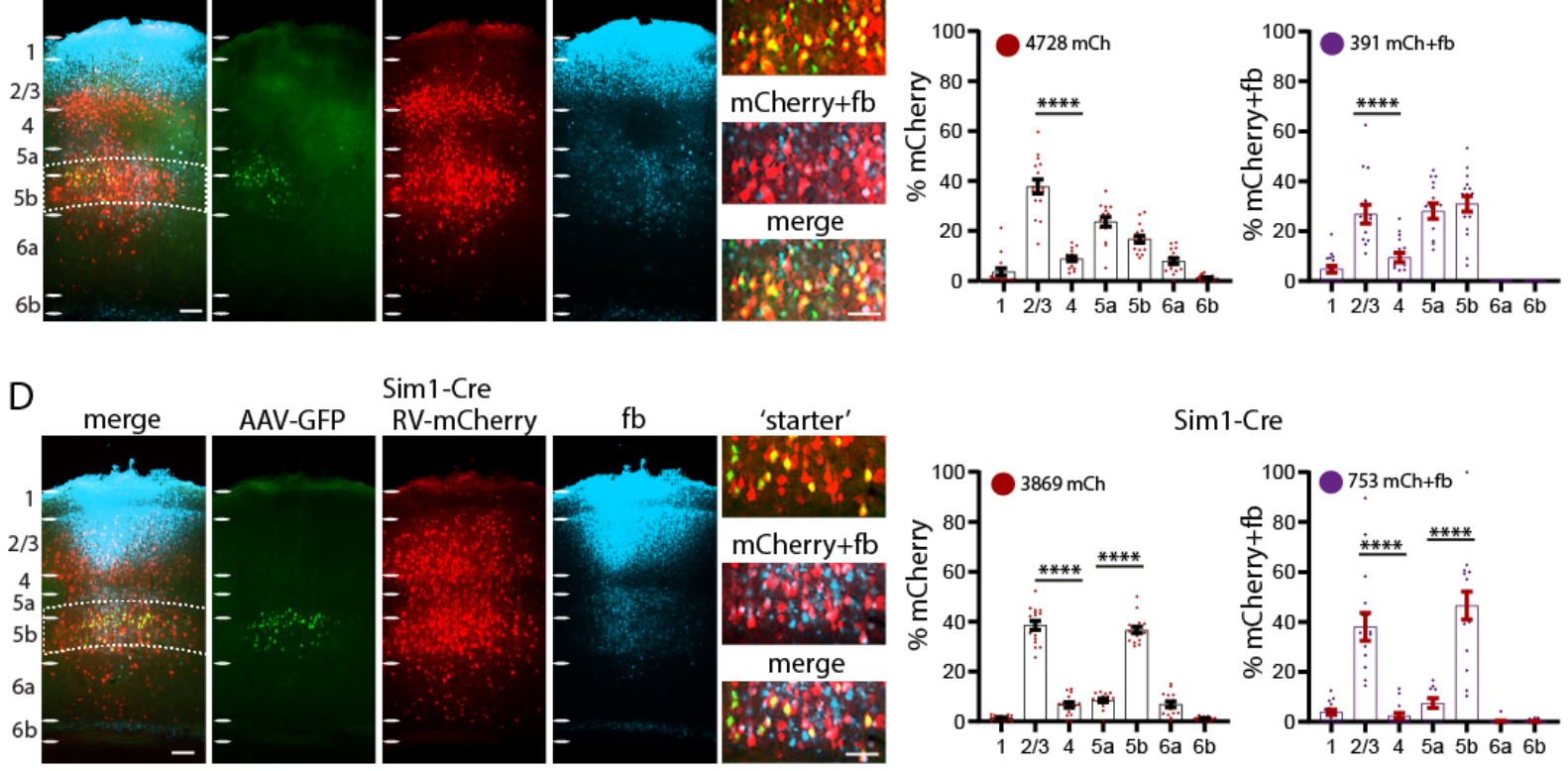

Sim1-Cre
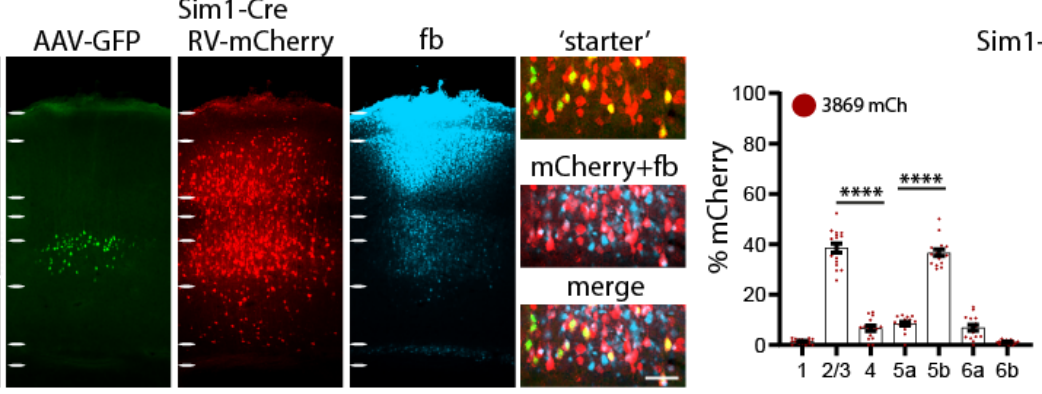

Sim1-Cre

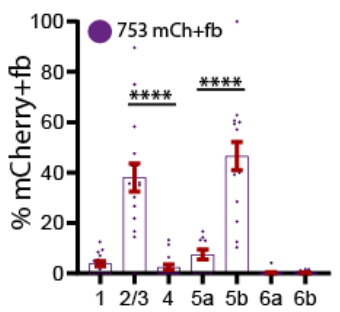

Figure 6. Local synaptic input to S1 L1 in Tlx3 and Sim1 neurons in combination with fb. (A) Schematic of injection scheme. Fourteen days after the first injection with AAV-Syn-flexnGToG-EGFP (step 1), fb was applied on L1 in parallel to the injection of rabies virus (steps 2 and 3). Both, rabies virus and fb were allowed to express for seven days. (B) Number of starter, presynaptic, fb labeled and double labeled neurons (presynaptic $+\mathrm{fb}$ ). In Tlx3-Cre mice, the presynaptic neurons derived from 479 starter neurons (out of 706 GFP expressing neurons), in Sim1-Cre mice the presynaptic neurons derived from 292 starter neurons (out of 643 GFP expressing neurons). (C, D) Example images of virus expression and fb uptake in S1 cortex of (C) Tlx3-Cre and (D), Sim1-Cre brains. Insets showing starter neurons, presynaptic neurons and fb double labeled neurons. Percentages of presynaptic neurons in S1 cortex and double labeled fb and rabies, neurons in Tlx3-Cre and Sim1-Cre brains Data shown as mean \pm S.E.M. Each dot in the graphs represents one brain section. Total number of neurons counted are shown in each panel. Statistical analysis with one-way ANOVA, Bonferroni post-hoc test, *** $\mathrm{p}>0.001$. Analysis details in Tables 6-1, 6-2. Scale bars in C, D $100 \mu \mathrm{m}$, in zoom-ins 50 $\mu \mathrm{m}$. 


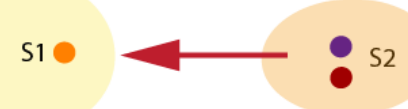

A
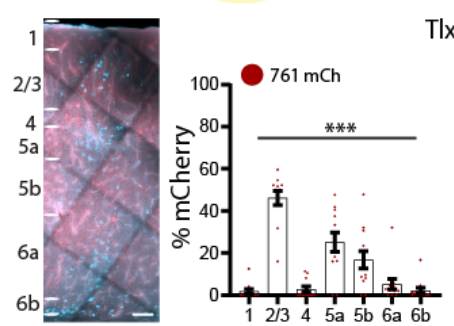

Tlx3-Cre

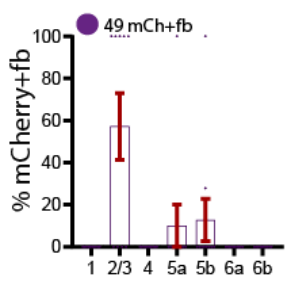

C
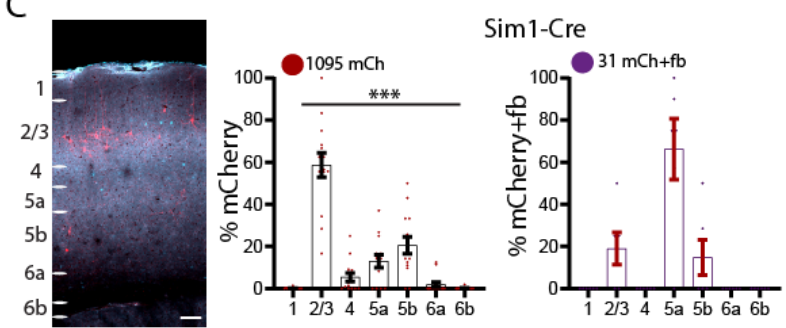

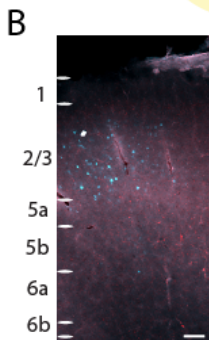

D
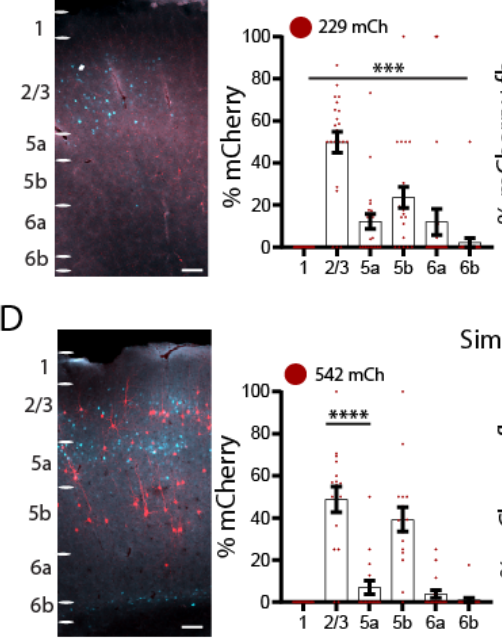

Tlx3-Cre

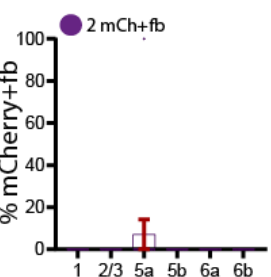

Sim1-Cre

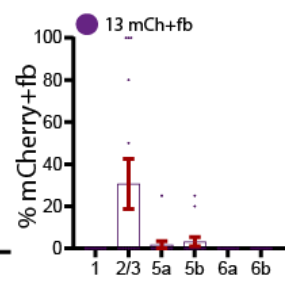

S1
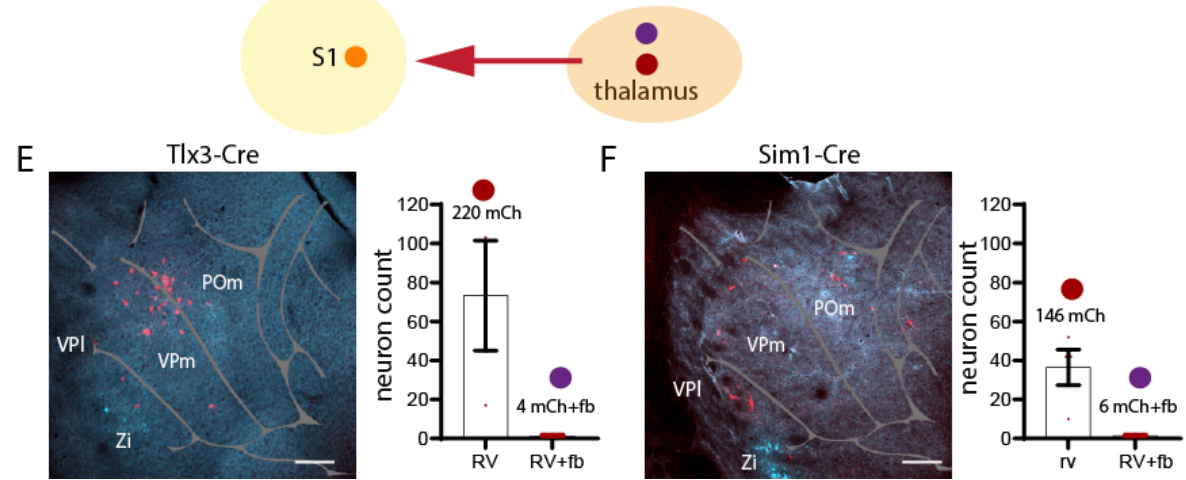

Figure 7. Long-range synaptic input to $\mathrm{S} 1 \mathrm{~L} 1$ in Tlx3 and Sim1 neurons in combination with fb. (A, B) Images and quantification of presynaptic neurons (rabies and fb double labeled) for Tlx3 brains in (A) S2 cortex ipsilateral, and in (B) motor cortices. (C, D) Images and quantification of presynaptic neurons (rabies and fb double labeled) for Sim1 brains, in (C) S2 cortex ipsilateral, and in (D) motor cortices ipsilateral. (E, F) Images of rabies expression and $\mathrm{fb}$ double label in thalamus in Tlx3 (E) and Sim1 (F) brains. Data shown as mean \pm S.E.M. Each dot in the graphs represents one brain section. Total number of neurons counted in each mouse line and brain are shown in each panel. Statistical analysis with one-way ANOVA, Bonferroni post-hoc test, ${ }^{* * *} \mathrm{p}>0.001$. Analysis details in Tables 6-1, 6-2. Scale bar in A-D, $100 \mu \mathrm{m}$, in E, F, $500 \mu \mathrm{m}$. 
A

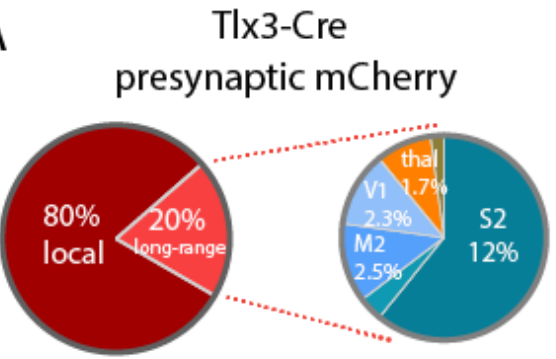

double labeled (fb+rabies)

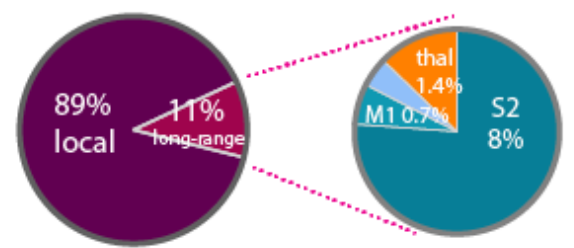

B

Sim1-Cre

presynaptic mCherry

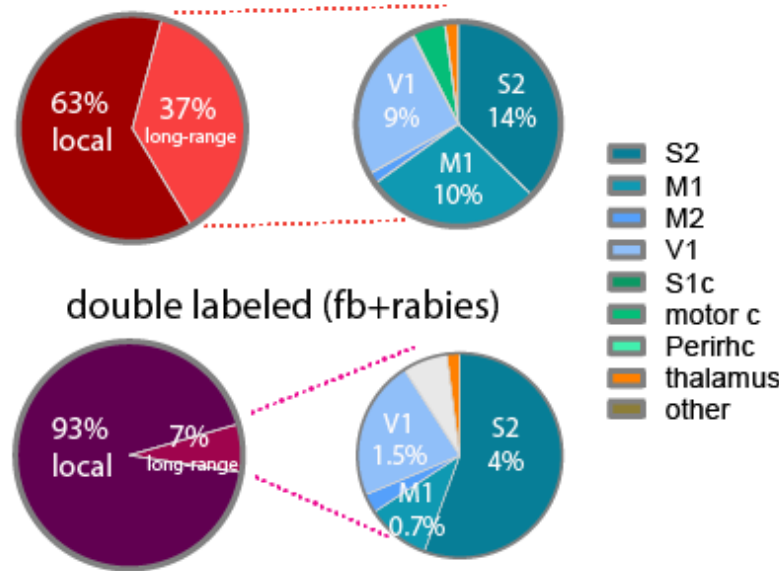

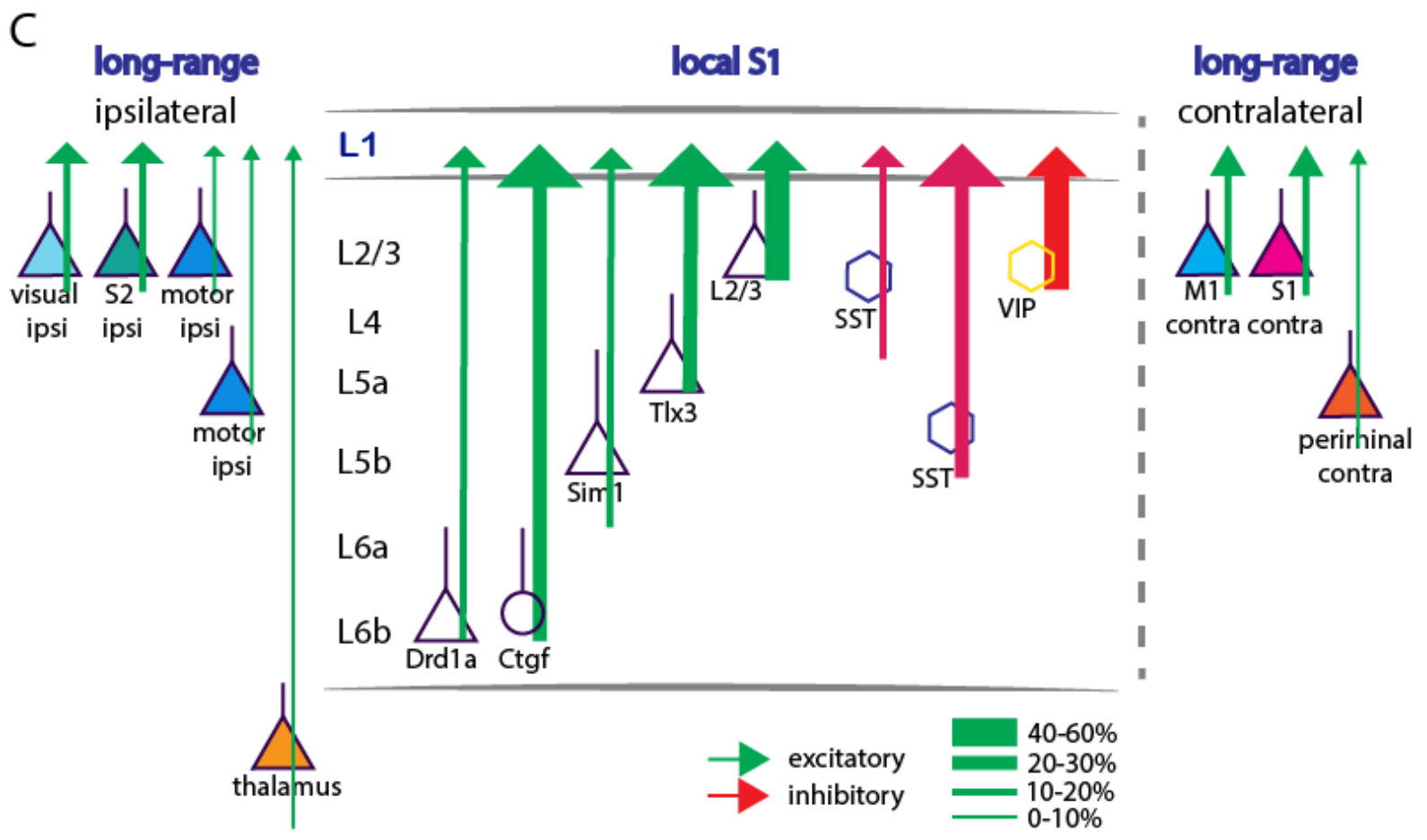

Figure 8. Summary of anatomical input to L1. (A, B) Pie charts showing presynaptic input to S1 for both IT and PT neurons (top) and pie charts showing L1 projecting neurons that are presynaptic to L5 neurons (bottom). Most input to IT and PT neurons was local. The longrange input to S1 L1 was from S2, V1, M1 and thalamus. For IT neurons the bulk of the neurons presynaptic to these cells that also projected to L1 were local neurons (89\%). Presynaptic input to PT neurons in S1 was divided into 63\% local and 37\% long-range. The long-range input was from S2, V1 and M1. Presynaptic input to PT neurons that also targeted L1 arose from local neurons (93\% of the total input). (C) Schematic of input to L1. Local input arose mainly from L2/3 and L5. Long-range input arose mainly from S2, motor and visual cortices ipsilateral to the application site and was less pronounced from the contralateral S1 and M1. The bulk of input from the perirhinal cortex was from the contralateral hemisphere. Analysis details in Table 8-1. 